\title{
Challenges in the Preparation of Optical Polymer Composites With Nanosized Pigment Particles: A Review on Recent Efforts
}

\author{
Mustafa M. Demir,* Gerhard Wegner
}

Blends of nanosized pigment particles and polymers are widely believed to offer the potential for the design of novel or at least improved materials. This review critically evaluates the recent literature with regard to the following issues: (a) why and how does the size of the particles matter, (b) what are the requirements to create compatibility between amorphous polymers and nanoparticles, (c) carbon allotropes as nanosized pigments, (d) bulk polymerization of monomer/pigment mixtures, (e) interaction of growing chains with the particles in the polymerization, (f) depletion flocculation as a mechanism to counteract homogeneous distribution of the particles in the polymer matrix and ways to suppress the undesirable flocculation, and (g) optical properties of the blends as well as methods of optical characterization.

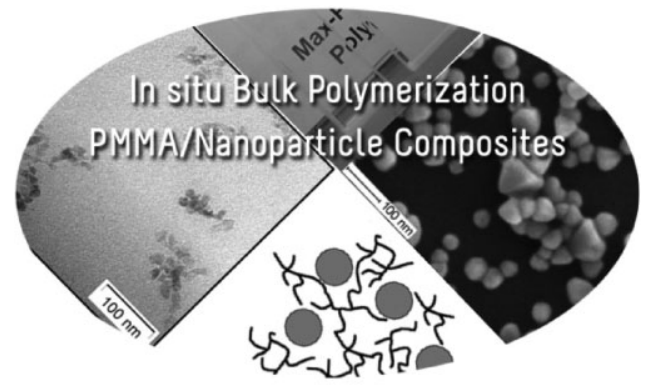

\section{Introduction and Scope}

The search for improvement of polymer performance by nanosized inorganic additives is well established since the 1990s when the term "polymer nanocomposite" began to evolve. According to the most accepted definition, the term polymer nanocomposites refers to multicomponent systems in which the major constituent is a polymer and the minor constituent has at least one dimension in the nanoscale regime $<100 \mathrm{~nm} \cdot{ }^{[1]}$ In 1993 , researchers of the Toyota Company claimed that adding minute amounts of clay platelets into nylon- 6 produced a fivefold increase in

Dr. M. M. Demir

Department of Chemistry, and Materials Science and Engineering Program, İzmir Institute of Technology, Gülbahçe Köyü, Urla 35430 Izmir, Turkey

E-mail: mdemir@iyte.edu.tr

G. Wegner

Max Planck Institute for Polymer Research, Ackermannweg 10,

D-55128 Mainz, Germany the yield and tensile strengths of the material. ${ }^{[2]}$ This study is considered as one of the stepping stones in the field of polymer nanocomposites. The number of related and follow-up studies has bloomed afterwards and it is currently one of the major interdisciplinary fields of science and technology including polymer science, materials science, nanotechnology, and biotechnology. However, the combination of polymers and inorganic particles was not a new event. Studies about blending of carbon black (CB) or "white" fillers such as ZnO or fumed silica with rubber fall into this category of research and have seen continuous developments since many decades. Automobile tire industry is one of the most important players in the field where the application of such materials is obvious. It is recalled that an automobile tire is essentially a blend of CB particles dispersed in heavily crosslinked rubber as the matrix. Polymer/color pigment systems are further examples for polymer nanocomposites. They are commonly found in coatings containing pigments and further heterogeneous additives like inhibitors and fire retarders, optimized for color, gloss, and corrosion protection. 
It is frequently said that the nanoscale size of additives opens a door for promising functional properties, which cannot be realized by micrometer sized traditional fillers. ${ }^{[3]}$ The main difference between composites prepared by conventional $\mu \mathrm{m}$-sized fillers and nanocomposites results from different states of particle dispersion. Let's assume that we observe the change of properties of a blend of a polymer at a fixed volume fraction of an inorganic additive ("pigment") than it is obvious that the number of particles per unit volume changes as $r^{-3}$ for spheres of radius $r$. The average of interparticle distance scales with $r$ assuming that the particles are homogeneously dispersed, and the surface/volume ratio changes as $r^{-1}$. For example, compare a conventional composite with a nanocomposite containing the same volume fraction of particles, where the spherical particles have $1 \mu \mathrm{m}$ and $10 \mathrm{~nm}$ in diameter, respectively. The total surface of the particles increases by two orders of magnitude, their number density increases by three orders of magnitude, and the interparticle distance diminishes by two orders of magnitude given that the particles are homogeneously mixed with the matrix polymer. Moreover, as the size of particles is reduced to nanoscale, the fraction of matrix volume belonging to the so-called excluded volume around each particle increases. The excluded volume considers many particle interactions. For example, the excluded volume comprises $5 \%$ of the nanocomposite system for spherical fillers of $10 \mathrm{~nm}$ radius at a loading of 1 vol\% assuming that the radius of excluded interactions amounts to twice the radius of the particle. In contrast, the excluded volume at this level of loading is negligible in conventional composite containing filler particles of 10-100 $\mu \mathrm{m}$ diameters. Hence, nanosized fillers allow to achieve property enhancement at lower loading levels compared to conventional fillers due to excluded volume effects. For instance, while the enhancement of, for example, mechanical properties of a neat polymer by incorporation of $\mu \mathrm{m}$-sized conventional inorganic fillers is first seen generally at loading levels in the range of 15$40 \mathrm{wt} \%$, the onset of changes is observed at lower loading levels between 1 and 5 wt\% with the same but nanosized filler. ${ }^{[1 a]}$ This difference in loading correspondingly determines the density of the resulting composite. Moreover, electronic and optical properties of certain materials, particularly semiconductors, can depend on size and shape of the nanocrystalline particles. The combination of such particles with polymers seems to offer a wide range of unique applications in electronics and photonics. ${ }^{[4]}$

With these general considerations and many claims from literature in mind the question arises as to: What is a "good" model system to experimentally study the properties of blends of nanosized particles and polymers. Of course, one may try to prepare (or obtain) the inorganic filler material in a particulate form and mix it with the desired polymer. However, the surface free energy of inorganics including

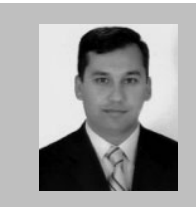

Mustafa M. Demir received his BS from Chemistry Department of Boğaziçi University, Turkey in 1999. He did his MS and PhD studies in Materials Sciences and Engineering Programme at Sabancı University in 2001 and 2004, respectively. Later he moved to Max Planck Society Polymer Research Institute in Mainz, Germany as post-doc researcher under the supervision of Prof. Gerhard Wegner. Since 2007, he has a faculty position at the Chemistry Department of Izmir Institute of Technology. His current research interest are polymer-based nanomaterials. He is now director of Materials Research Center and coordinator of Materials Science and Engineering Program of the Institute.

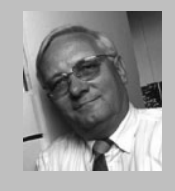

Gerhard Wegner joined the Max Planck Society as one of the founders of the Max Planck Institute for Polymer Research in Mainz. He holds a doctoral degree in chemistry from the University of Mainz (1965). In 1966-1969 he worked as a research staff chemist at Yale University, Conn., USA. Returning to the University of Mainz, he joined the group of Prof. E. W. Fischer in the Institute of Physical Chemistry. His habilitation in physical chemistry (1970) concentrated on solid-state polymerization of diacetylenes and showed how macroscopic single crystals of polyconjugated macromolecules can be made. From 1974 to 1984 he held the chair in Macromolecular Chemistry at the University of Freiburg (Germany). He has received numerous awards, including the Hermann Staudinger Medal of the German Chemical Society (1990), the ACS Award in Polymer Chemistry (1998), and the Award of the Society of Polymer Science Japan (1998). He served as the chairman of the Chemistry-Physics-Technology section of the Max Planck Society from 1991 to 1994 and was Vice President of the Max Planck Society from 1996 to 2002. Professor Wegner is also the founder and chairman of the current International Max-PlanckResearch School for Polymer Science (IMPRSPMS) and serves as its chairman as well as for the Early Stage Training (EST)-Marie-Curie-Action.

carbonaceous materials is quite large, typically in the range of hundreds or thousands of $\mathrm{mJ} \cdot \mathrm{m}^{-2}$ and for polymers it is at least one order of magnitude lower $\left(20-50 \mathrm{~mJ} \cdot \mathrm{m}^{-2}\right){ }^{[5]}$ This difference in surface energies leads to segregation of the inorganic particles, namely formation of agglomerates of grains which are usually extremely difficult to break up into individual species. Wetting of the individual particles by the polymer segments is unfavorable against particle/ particle interaction for enthalpic reasons even if individual particles were present. The extent of wetting of materials components, in this case particles with polymers is defined as compatibility that can be improved modifying the

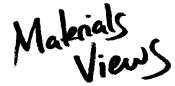

www.MaterialsViews.com 
surface chemistry of the particles with organophilic surfactants.

In the further text, regions in the polymer matrix containing the dispersed inorganic particles are called domains. A domain contains either one individual particle or an assembly of individual particles. Depending on the size of domains and the extent of interaction between individual particles, the assembly could be an aggregate/ agglomerate or a cluster of particles. Although these terms are related to each other and have been frequently used interchangeably, they differ in morphological details with regards to the nature of particle dispersion. In an aggregate, individual particles are tightly associated with each other such that they form a compact body which cannot be easily redispersed into individual particles. Agglomerate is an assembly of aggregates linked by weak interactions, so that it can be reduced to the size of aggregates by physical means. On the other hand, clusters are composed of loosely associated individual particles and/or aggregates. They are defined as particle-rich domains meaning that at least a minute amount of polymer chain segments is present in between the individual particles. These polymer chains hold the individual particles together and also prevent tight association. Individual particles in a cluster can be redispersed when they are isolated from the surrounding polymer matrix. These differences in the nature of particle dispersion determine the macroscopic performance of the nanocomposite material.

An important physical property achieved as the size of particles is reduced to the nanoscale is transparency. When the size of individual particles becomes much smaller than the wavelength of visible light (e.g., $0.5 \mu \mathrm{m}$ ), the particles contribute less to scattering of light and, under certain circumstances, transparency can be achieved when nanoparticles and amorphous polymers are blended. However, the aggregation/agglomeration of primary particles is unavoidable in many cases. Due to the strong scattering power of large aggregate/agglomerate structures, it remains a big challenge to achieve transparency for the more interesting polymer/particle systems. However, one needs to recall that nature exhibits excellent transparency in certain complex biological tissues. For example, the Cornea, the front cover of the eye, is an optically clear material that focuses light into the retina and serves a significant function in image formation by the eye. It is comprised of randomly oriented stacked sheets called lamella containing unixially aligned collagen fibrils embedded in an optically homogeneous ground substance (proteoglycans). The corneal fibrils are very long and of uniform thickness of nearly $20 \mathrm{~nm}$ in diameter. They are distributed throughout the matrix at fixed distance. Smallangle X-ray scattering (SAXS) measurements showed that the constant spacing between all fibrils is caused by proteoglycans which are covering each collagen fibril. ${ }^{[6]}$
Despite the considerable effort that has been put into understanding of the structure of the cornea, there is still no universally accepted explanation to the transparency of this heterogeneous material. ${ }^{[7]}$ Over the years, many models have been proposed. According to the commonly used one put forward long ago by Maurice, ${ }^{[8]}$ the visual acuity of this heterogeneous material is a result of the packing of fibrils in a periodic arrangement. Their relative positions lead to destructive interference of light scattered away from the forward direction, all the light intensity contributing to the constructive interference in the forward direction. Hence, the overall scattering of hierarchically ordered collagen fibrils in the cornea is always smaller than total scattering of individual fibrils. The arrangement of the fibril' axes in the stroma may be described quantitatively in terms of a radial distribution function. It specifies the likelihood of finding two fibril axes separated by a given distance and characterizes the homogeneity of the fibril dispersion in the proteoglycan matrix. It was demonstrated that the collagen fibers exhibit random displacements from their ideal positions in a perfect lattice and that a short range order rather than a long range periodic placement describes the situation best. ${ }^{[9]}$

Motifs exhibited by natural tissues like in the cornea of the human eye can be a source of inspiration for material scientists to design new synthetic materials. ${ }^{[10]}$ As shown for this particular example, internal structure plays a key role in optical performance of composite materials. For polymer/particle composites, the control over internal structure is mainly a matter of the preparation methods employed. There are principally three different methods to fabricate polymer nanocomposites. In the first one, nanoparticles (NPs) are prepared separately and they are subsequently blended with a desired polymer either in solution or in the melt. ${ }^{[11]}$ However, NPs tend to suffer from agglomeration and - even worse - aggregation during handling and/or storage, which, in turn, gives rise to an inhomogeneous distribution of particles within the polymer matrix. Therefore, another method tries to avoid the isolation of the nanoparticles by preparing them in situ. ${ }^{[12]}$ In other words, nucleation and growth of the NPs occur in the presence of the host matrix polymer. Since the viscosity of polymers is comparatively high and hampers their diffusion, the resulting NPs are prevented to undergo aggregation for reasons of kinetics. The major disadvantage of this procedure is that the resulting polymer composite remains contaminated by unreacted educts or byproducts of the formation of the NPs. The third method involves bulk polymerization of a suitable monomer in the presence of NPs. It has certain advantages for the following reasons. An additional organic solvent is not needed. The monomer serves as a medium to disperse the colloidal NPs. Once polymerization has occurred, no further purification from the solvent is necessary provided that polymerization can 


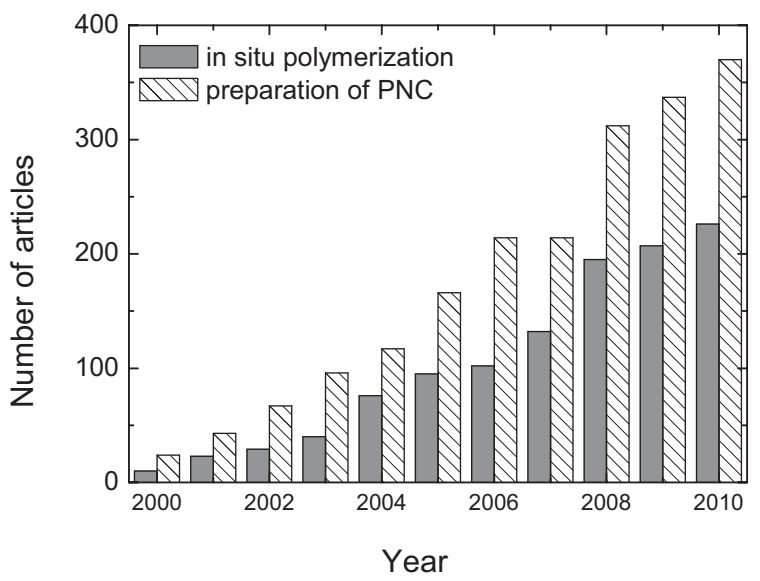

Figure 1. The number of articles for "preparation" (in general) and "in situ polymerization" of polymer nanocomposites per year in Web of Science database. The result of this statistical study shows the importance of in situ polymerization process in the preparation of polymer nanocomposites.

be driven to completion. Since 2000s and especially in recent years, the in situ polymerization process has gained more attention probably due in part to a surging interest in nanotechnology because homogeneous polymer/nanoparticle blends can be easily fabricated with this process. A survey of open publications related with in situ polymerization in the past 10 years is given in Figure 1. These literature data were obtained based on a Web of Science Scholar search system. The data clearly demonstrated that the preparation of polymer nanocomposites has attracted increasing attention recently, and majority of the fabrication process has been carried out by in situ polymerization. Strangely enough, although the process has shown potential promising and has existed in the literature, its understanding is still very limited. In this paper, a systematic review is made on the process related to fabrication of polymer nanocomposites including structure and optical property characterization. Other issues regarding the technology limitations, research challenges, and future trends are also addressed in the paper.

A fourth possibility in preparation of polymer nanocomposites is a mixed method of in situ polymerization and in situ preparation of nanoparticles. In situ sol/gel synthesis falls into this category. ${ }^{[13]}$ Here, both polymerization and particle precipitation occur in situ and simultaneously. Considering the occurrence of two dissimilar synthetic reactions in one pot, one is tempted to assume that the purity of both nanoparticles and polymers will be seriously affected in this approach. A recent example serves to illustrate the points. Toprak and co-workers ${ }^{[13 b]}$ reported the formation of poly(methyl methacrylate) (PMMA)/ZnO composites by such an approach. The particle precursors zinc acetate dihydrate and monoethanol amine were dissolved in the monomer of interest, methyl acrylate (MMA). When the temperature of the mixture was increased, zinc acetate dihydrate became hydrolyzed. Simultaneous with nucleation and growth of the thermal polymerization is initiated. Eventually, a solid-composite of PMMA and ZnO NPs is formed. However, it is well known that hydrolysis of $\mathrm{ZnO}$ starting from zinc acetate is a highly complex reaction since zinc hydroxy acetate forms as a rather stable intermediate and, therefore, it is hard to obtain pure $\mathrm{ZnO}$ as the final product. ${ }^{[14]}$ Similarly, the interactions of propagating free radicals with the monomer/polymer/ surfactant mixture is unknown and, therefore, control of molecular weight and molecular weight distribution of the matrix polymer remains an unsolved and unidentified problem. Consequently, the product obtained via this strategy is ill defined.

Atactic PMMA is an amorphous transparent thermoplastic, often used as a light weight and shatter-resistant alternative to glass. ${ }^{[15]}$ Its density is less than half of that of window glass. It transmits light in the range of 360$1000 \mathrm{~nm}$ almost without loss, meaning that it exhibits a transparency of $92 \%$ at normal incidence (similar to window glass) with a high surface gloss. Its refractive index (RI) is 1.49 at $589 \mathrm{~nm}$. It is extraordinarily resistant to oxidative photo degradation and therefore remarkably stable to sunlight. PMMA is therefore often the material of choice for outdoor applications. It finds use for many applications, for example, lenses, ${ }^{[16]}$ reflectors, ${ }^{[17]}$ intraocular lenses, ${ }^{[18]}$ cores of communication grade polymer optical fibers, ${ }^{[19]}$ and also as substrate for polymer optoelectronic devices $^{[20]}$ such as materials to fabricate integrated waveguides. PMMA has been identified as the material of choice for light guiding structures in the back illumination of liquid crystal displays (LCDs). ${ }^{[21]}$ It has favorable processing properties $\left(T_{\mathrm{g}}=105^{\circ}\right)$, and can be modified with pigments, UV light absorbing additives, and scratch resistant coatings. Hence, it has been frequently used as a host matrix for various pigments to obtain optical functionality.

This paper reviews recent work on preparation of PMMA nanocomposites with various nanosized pigment particles. Since the main method for mass production of PMMA is free radical bulk polymerization, the primary focus is placed on free radical polymerization of MMA carried out in the presence of nanosized pigment particles.

This paper is organized as follows: after an introduction providing motivations and scope (Section 1), every step of the preparation process including fabrication of nanoparticles with a well-defined surface chemistry (Section 2), the effect of the particles on the polymerization process in terms of microstructure, conversion, and molecular-weight distribution (MWD) of polymer chains (Section 3), and the influence of topology of in situ formed chains on the particle

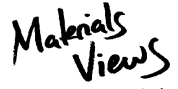

www.MaterialsViews.com 
dispersion (Section 4) is outlined separately. In Section 5 , the optical properties of the nanocomposites are discussed. The last section (Section 6) concludes the document and summarizes the open challenges.

\section{Nanoparticles for Dispersion in Polymers}

In the last three decades, a diverse spectrum of pigments of nanoscale, that is, colloidal dimension including carbonaceous, metal, metal oxide, and chalcogenide particles have been combined with polymers. The variations in particle chemistry allow developing tailor-made functional materials for various applications. As far as optical properties are concerned, blends with carbonaceous particles [carbon black (CB), graphenes, and fullerenes including carbon nanotubes (CNTs)] have been thoroughly studied. In particular, CB in its various qualities is and has been commonly used as a pigment since the very earliest times of polymer technology. ${ }^{[22]}$ Today, it is found in photocopier and laser printer toners as well as in automobile tires. While the hiding power and optical contrast is the reason for the former application, it is the strong improvement of the mechanical relaxation behavior combined with protective properties against solar radiation induced damages in the latter which stands behind application. CNT and fullerenes are still unconventional pigments compared to CB. They are carbon allotropes of different dimensionality at nanoscale. CNTs are one-dimensional objects that are fibrils of very large aspect ratio. Problems arising when it is attempted to fabricate blends have been thoroughly reviewed literature. The zero-dimensional carbon allotropes, fullerenes, can be envisioned to be made by wrapping sections of a graphene sheet. ${ }^{[23]}$ They have raised expectations for their potential to be used in applications where electronic properties need to be tailored or optimized, for example, in solar cells. NPs of noble metals like gold or silver have been studied as pigments for reasons of their specific optical properties. They exhibit a strong absorption maximum at specific wavelengths due to photon-induced oscillation of surface electrons (surface plasmons). The absorption and excitation profile can be tailored by size and shape of the particles. ${ }^{[24]}$ Significant red shift and broadening of the surface plasmon has been observed upon incorporation into polymeric matrices. ${ }^{[25]}$ According to the Drude model, the plasmon resonance frequency is expected to respond to the dielectric medium of the surrounding polymer. ${ }^{[26]} \mathrm{A}$ broadening of absorption and red shift have been seen for many cases in which polymers were the matrix for the dispersion.

$\mathrm{SiO}_{2}$ is a commonly used filler for polymers. ${ }^{[27 \mathrm{a}]}$ Fumed silica is an amorphous, non-porous form of silicon dioxide $\left(\mathrm{SiO}_{2}\right)$ with a very low density and a fluffy nature. ${ }^{[27 \mathrm{~b}]}$ (Figure 2) It has a lower RI compared to metal oxides; therefore, it is easier to achieve a RI matching with

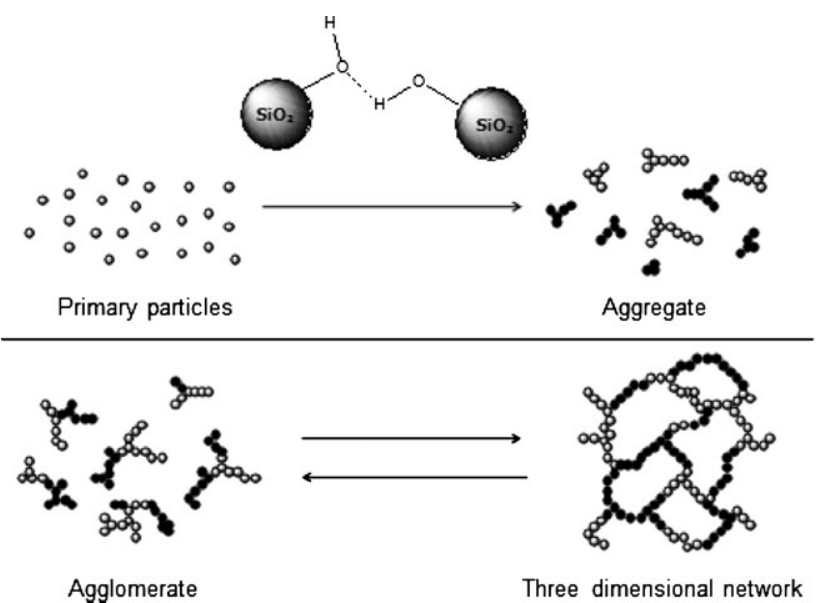

Figure 2. Schematic representation of the formation of aggregates, agglomerates, and networks of fumed silica particles.

polymers. Adding silica nanoparticles to polymers improves scratch resistance and other mechanical properties of polymers. ${ }^{[28]}$ The native surface of silica exhibits siloxane and silanol groups. The latter are prone to form hydrogen bonds either with solvent, residual water, or other silanol groups of closely spaced silica nanoparticles. Particularly the latter interaction enhances the particle connectivity. ${ }^{[2]}$ These silanol groups can be replaced by other functional groups, so that interactions between particles or particle and medium can be tuned. Primary particles are produced on industrial scale in the nm-range and with a narrow size distribution; however, they have the tendency to fuse together forming stable aggregates in the size range of $0.1-0.5 \mu \mathrm{m} .{ }^{\left[{ }^{30]}\right.}$ Since interparticle forces are non-directional, the primary particles may be locked into configurations such that the aggregates are not dense. Indeed, many colloidal silica aggregates have structures that are bushy, dendritic, or fractal. Interparticle hydrogen bonding has been shown to be the key factor controlling the stability of dispersions and is the origin of network formation. When silica particles are dispersed in a medium, for instance polymers, aggregates will form that can span across the entire volume and create mechanical connections between remote points of the polymeric structure, thereby controlling the rheo-mechanical properties. ${ }^{[31]}$ The network structure within a medium can be particularly extensive under stress-free conditions. When the aggregates are submitted to large external forces such as mechanical compression or shear forces, these forces may cause the aggregates to break up, collapse, or reform in other ways. Consequently, dispersions of fumed silica show a solid-like rheological response and the response of the aggregates to applied forces may determine how they perform in given applications. ${ }^{[32]}$

Metal oxides $\left(\mathrm{TiO}_{2}, \mathrm{ZnO}\right.$, and $\left.\mathrm{CeO}_{2}\right)$ are an important group of pigments used in coatings, insulators, cosmetic 
materials as well as building materials, for example, window frames, and as photocatalysts. They are transparent over the whole visible and IR region of the spectrum; however, they are absorbing in the UV range. For example, $\mathrm{TiO}_{2}$ is a wide band gap ( $3.15 \mathrm{eV}$ ) semiconductor absorbing light below $380 \mathrm{~nm}$. $\mathrm{TiO}_{2}$ exists in several polymorphs; the solid powderous material is commonly a mixture of anatase and rutile. The rutile phase is obtained uniquely at high temperatures $\left(\approx 600^{\circ} \mathrm{C}\right)$ while the commonly observed phase is anatase if $\mathrm{TiO}_{2}$ is prepared above or near at room temperature. The use of $\mathrm{TiO}_{2}$ particles toward fabrication of transparent nanocomposites is problematic because it is nearly impossible to individualize the nanoscale crystals from their aggregates which are inevitably formed in their preparation. However, these oxidic particles are frequently used with polymers not only as UV shielding pigments but also as agents for photocatalytic oxidations. ${ }^{[33]}$

$\mathrm{ZnO}$ is a convenient pigment possessing only one crystalline phase, namely zincite. It is a non-toxic wide bandgap $(\approx 3.3 \mathrm{eV})$ semiconductor that absorbs near UV light and emits in the visible region. The emission is related to the presence of oxygen defects in the zincite lattice. $\mathrm{CeO}_{2}$ with band gap of $3.55 \mathrm{eV}$ is also a very attractive material as an UV light absorber. ${ }^{[34]}$ A further interesting material is, $\mathrm{SnO}_{2}$, a highly conductive and transparent material. Impurity doped $\mathrm{SnO}_{2}$ has been found to be more effective in conduction and light absorbing power than pure $\mathrm{SnO}_{2}$ itself. $\mathrm{SnO}_{2}$ doped with antimony or indium is used to induce efficient absorption in the IR region of the electromagnetic spectrum. This feature is particularly interesting for commercial applications in integrated optical devices like fiber amplifiers and for waveguide telecommunication. ${ }^{\text {[35] }}$

In addition to metal oxide particles, chalcogenides (sulfides, selenides, and tellurides) have been frequently used as pigments in nanocomposites aiming for optical materials. ${ }^{[36]}$ They are interesting for their strong emission features in the visible region. They exhibit size-dependent UV-visible emission and absorption. Moreover, it is relatively simple to control the shape of the powder particles. For instance, octadecyl- $p$-vinylbenzyldimethylammonium chloride-coated CdTe particles presented emission in the green for a given diameter of $2.8 \mathrm{~nm}$, in the yellow for a diameter of $3.3 \mathrm{~nm}$, in the orange for a diameter of $3.6 \mathrm{~nm}$, and in red for a diameter of $4.0 \mathrm{~nm}^{\text {[37] }}$ Polymers loaded with such particles systems are considered as novel materials and components of light emitting diodes and in photovoltaics. ${ }^{[38]}$ It has been claimed that nanosized pigments of this type exhibit higher stability and better quantum efficiency in luminescence compared to organic materials under the same or similar conditions of applications. ${ }^{[39]}$

Much attention has been devoted to the synthesis of different chalcogenide nanocrystals and to their incorporation into polymer matrices. The most commonly used chalcogenides in the context of polymers are $\mathrm{CdSe}^{[40]}$ $\mathrm{CdS},{ }^{[40]} \mathrm{CdTe},{ }^{[41]}$ and $\mathrm{PbS} .{ }^{[42]}$ However, the toxicity and high cost of $\mathrm{Cd}-$, Se-, or As-containing compounds have an adverse effect on the potential of these materials for practical application. Serious attempts are made to address this issue by developing highly luminescent nanocrystals which do not contain toxic elements. ${ }^{[38 a, 43]}$ For instance, $\mathrm{ZnS}$ is a non-toxic, chemically stable wide band gap $(3.8 \mathrm{eV})$ semiconductor. It has been incorporated into various polymeric media in form of nanopigment. ${ }^{[36 a]} \mathrm{ZnS}$ is also used as the passivating shell to suppress the diffusion of toxic elements from inside the core of the particles. The development of core/shell-type nanostructured pigments like CdSe/ZnS ${ }^{[44]}$ and $\mathrm{CdS} / \mathrm{ZnS}^{[45]}$ is well established. It is also worth mentioning that the emission features of $\mathrm{ZnS}$ pigments can be enhanced by doping with manganese and similar elements. ${ }^{[38 a]}$

\subsection{Surface Functionalization of Nanoparticles}

A major obstacle preventing the formation of homogeneous nanocomposites arises from the poor compatibility of the two dissimilar constituents. Surface modification of particles is crucial to attain compatibility of the nanoparticles with the polymer matrix. In general, there are two approaches for surface modification of a solid by polymers: grafting to and grafting from. In the former, a surfactant (small molecule or polymer) contains anchoring groups which will bind to the particle surface. By reaction of these groups with functional sites, for example, surface $-\mathrm{OH}$ groups on the particle they will be grafted to the inorganic particle. This approach is restricted to low grafting densities because of steric hindrance imposed on further reaction by already grafted chains. In the case of "grafting from" initiating groups have to be attached to the surface which allow polymerization radially outward from the surface. This approach provides for brush-like dense surface structures with controllable graft density. In both approaches, the selection of a proper surfactant is of utmost importance as they adsorb to surfaces in a substrate specific manner. Silane coupling agents, as example, are frequently used to functionalize the surfaces of oxides of silicon, aluminum, zirconium, tin, titanium, and nickel. Less stable bonds can be formed with other oxides. ${ }^{[46]}$ Moreover, thiols are excellent to create stable contact with gold, silver, and chalgogenides owing to formation of strong metalsulfur bonds. Pt and Pd are capped with amines, or alkyl cyanates; metal oxides can also be surface modified by acidic surfactants. ${ }^{[4]}$ Certain biogenic molecules like lysine or fructose were found to be efficient surface active materials in this context. ${ }^{[48]}$ Fructose was shown to be quite beneficial to achieve good dispersion of $\mathrm{Al}_{2} \mathrm{O}_{3}$ particles. ${ }^{[49]}$ In addition to small surfactant molecules, polymers, for example, amphilic copolymers have also been

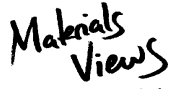

www.MaterialsViews.com
Macromol. Mater. Eng. 2012, 297, 838-863

(c) 2012 WILEY-VCH Verlag GmbH \& Co. KGaA, Weinheim 
used to functionalize the particle surface. For example, Mullen and co-workers ${ }^{[50]}$ have prepared a surface-active polymer via free radical copolymerization of lauryl methacrylate (LMA) and 2-(dimethylamino)ethyl methacrylate (DMAEMA). This copolymer was reacted with 1,3propanesulfonate so that ionogenic groups on the polymer backbone were formed. The authors claim that the ionic groups adsorb to the $\mathrm{ZnO}$ particle surface by a large number of binding sites and the remaining hydrophobic chain segments improve the compatibility with PMMA matrix. Following their conclusions particle domains of sizes smaller than $100 \mathrm{~nm}$ were obtained. ${ }^{[50]}$ However, it must be noted that a strong phase separation takes place between the bulk polymer and the polymer chains firmly attached to the particle surface. Such phase separation is undesirable in that it can strongly reduce the performance of the material. A similar approach was proposed for a process by which $\mathrm{SiO}_{2}$ nanoparticles were to be transferred from an aqueous dispersion into a non-polar polyurethane (PU) matrix to obtain homogeneous particle dispersions free of aggregates. ${ }^{[51]}$

\subsubsection{Controlled Chemical Precipitation}

In any application of nanosized particles involving fluids of low viscosity as the medium of dispersion spontaneous sedimentation is an obvious problem. This problem is well known in the coating and paint industry from where a number of recipes are known to counteract this phenomenon. Of course, the rate of sedimentation under the influence of gravity depends on the mass and hydrodynamic volume of the particles. Brownian motion counteracts sedimentation. Therefore, the best recipe to avoid the undesired effects of sedimentation is to create as small as possible particles and provide them with a non-interacting surface coating that prevents bridging among individual particles. However, under certain circumstances it is desirable to create nanoparticles with a specific but weak potential for mutual interactions such that clusters and eventually networks of clusters can be formed which can be reversibly destroyed and, eventually, reformed depending on the magnitude of applied mechanical forces. A wellknown example are silica nanoparticles which are employed as thickeners in the formation of thixotropic paints and printing inks (compare for Figure 2). These considerations are particularly important if one wants to carry out polymerizations of dispersions of nanoparticles in the bulk monomer. Depletion of fractions of the total reaction volume from particles must be prevented in order to obtain a homogeneous particle density over the whole volume of the object that is created by bulk polymerization, for example, a thick sheet. Of course, a process must be at hand which allows fabricating the required nanoparticles in sufficiently large quantities under strictly reproducible conditions. Otherwise reproducible fabrication of a required number of large enough objects to allow for tests of mechanical, optical, electrical, etc. characterization is not possible, not to speak about pilot plant or even larger production of parts. In other words any process suggested for the production of nanoparticles must have the potential to allow up scaling at least to the kg-range and/or translation from a batchwise to a continuous process of production.

In a typical synthesis where particles are created in a solvent by reaction between soluble reactants at a temperature much below the solubility product of the precipitating solid phase one needs to differentiate between nucleation of the particles, their growth, and their ripening. Following the traditional picture, nucleation is highly temperature dependent. It can occur homogeneously as a spontaneous process or it can be controlled by a nucleating agent in which case we speak about heterogeneous nucleation. Furthermore, one needs to differentiate between athermal and thermal nucleation. In the latter case particles are nucleated continuously over the full length of the particle synthesis. This will naturally lead to a very broad distribution of particle sizes since particles which were nucleated at the beginning of the precipitation process have longer time available for growth as compared to particles which have been created at later time. In the case of so-called athermal nucleation all particles are created within a short period of time in the initial phase of the precipitation process, and the barrier for further nucleation is high enough so that only growth of the already formed particles is possible. Therefore, a specific population of particles will grow which exhibits a narrow size distribution. However, a size distribution among growing particles will inevitably lead to ripening, that is, mass transfer between smaller and larger particles, this process being driven by differences in the free energy between the particles of different sizes. In other words small particles disappear in favor of larger ones. ${ }^{[52]}$ This will lead to a continuous change of the size distribution as long as the particles are in contact with each other in the reaction medium. Of course, other modes of interactions are possible and are frequently experienced. One of these is agglomeration among particles by contact followed by bridging via physical or chemical bonds. Agglomerates are composed of a number of individual particles, for example, nanosized crystals held together by the secondary forces. ${ }^{[53]}$ These can be so strong that it becomes a difficult task to individualize the component nanosized particles.

In the light of these considerations, it is advantageous to develop synthetic procedures under which one is able to create conditions for athermal nucleation of the desired particles followed by a short growth period in which the desired size can be obtained without inference from ripening features as to create a narrow size distribution. [54] Finally a capping reaction needs to be applied which 


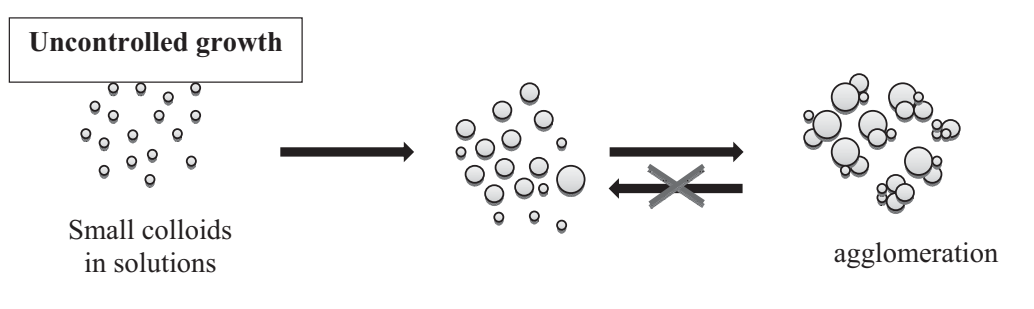

Controlled growth

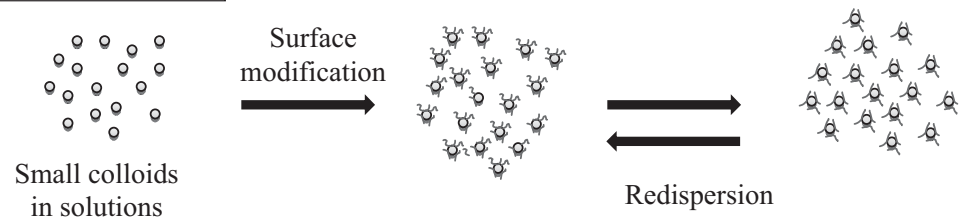

Figure 3. Sketch exemplifying the meaning of "uncontrolled growth" of individual particles followed by agglomeration (above) and "controlled growth" (below), the latter leading to redispersible particles of narrow size distribution. Reproduced from ref. ${ }^{[55]}$ by permission of The Royal Society of Chemistry.

suppresses further growth of the particles, and at the same time modifies the particle surface in a manner to define its surface energy and, thereby the wetting behavior with regard to organic solvents and/or polymers.

These requirements can be met by controlled chemical precipitation. When certain metal cations and suitable anions are combined in a supersaturated solution, homogeneous precipitation occurs and insoluble solid particles are produced. Their surface may be protected in situ by reaction with a capping agent. A schematic demonstration of this process is depicted in Figure $3 .^{[55]}$

The optimum time for obtaining the smallest particle size is right after nucleation. As an example, Figure 4a shows the number size distribution of tin(IV) oxide nanoparticles obtained by controlled precipitation using tert-butylphosphonic acid $\left(t \mathrm{BuPO}_{3} \mathrm{H}_{2}\right)$ as capping agent in MMA. ${ }^{[56]}$ The synthesis of these particles is based on esterification of tin(IV) acetate with 1-pentanol under reflux. A kinetic study indicated that the nucleation of the tin oxide occurs approximately within $1 \mathrm{~h}$ of reaction time under these conditions. The capping agent adsorbs to the surface of growing crystals and suppresses the growth of particles. When the capping agent was added after $4 \mathrm{~h}$, the particles had more time for growth and their average diameter was $177 \mathrm{~nm}$. When it was added after $3 \mathrm{~h}$, the diameter was only $110 \mathrm{~nm}$. Generally speaking, the particle size was smaller when the capping agent was added earlier, and ageing of the surface-modified particles did not cause a detectable increase in the size of the nanoparticles in this process. ${ }^{[57]}$ In other words, ripening is efficiently suppressed by the capping agent and this demonstrates the importance of finding suitable capping agents.

It is important to know about the area density of capping moieties per particle in addition to particle size and particle size distribution (PSD). A further example refers to the production of $\mathrm{ZnO}$ nanoparticles. They were obtained by a similar process, namely reaction of zinc acetate dihydrate with 1-pentanol. ${ }^{[14]}$ The amount of capping agent with respect to the metal oxide precursor in terms of molar ratio P:Zn was varied. ${ }^{[58]}$ Figure $4 \mathrm{~b}$ presents number/size distributions of the neat particles from dynamic light scattering (DLS) and the particles treated with different amounts of $t \mathrm{BuPO}_{3} \mathrm{H}_{2}$ and tetrabutylammonium acetate (TBAc, cocatalyst). While the unmodified particles exhibit $\mu \mathrm{m}$ scale diameters and broad size distribution and in fact are agglomerates, the ones treated with $t \mathrm{BuPO}_{3} \mathrm{H}_{2}$ turn out to be individual particles with average diameters of $22 \mathrm{~nm}$ and a size distribution with a short tail ending at around $80 \mathrm{~nm}$ at $\mathrm{P} / \mathrm{Zn}=0.34$. Analysis of the surface-modified particles shows that the capping agent results in monolayer coverage on the particle surface. The amount of capping moieties per unit area is proportional to the initial
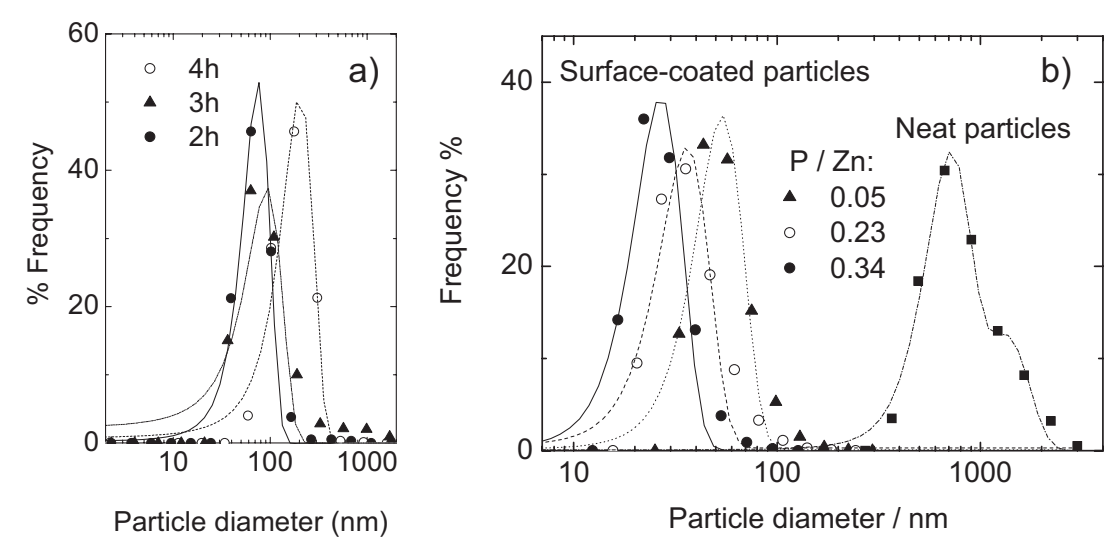

Figure 4. (a) Size distribution (determined by $\mathrm{DLS}$ ) of $\mathrm{SnO}_{2}$ particles dispersed in MMA. The particles were obtained by controlled precipitation at reaction times of $5 \mathrm{~h}$ in total with addition of $\mathrm{tBuPO}_{3} \mathrm{H}_{2}$ as capping agent after 2, 3, and $4 \mathrm{~h}$ reaction time. Data reprinted from ref. ${ }^{[56]}$ (b) Size distribution of $\mathrm{ZnO}$ particles in their "neat" form and of the same particles surface capped with $\mathrm{tBuPO}_{3} \mathrm{H}_{2}$ at different degrees of surface coverage ( $\mathrm{P} / \mathrm{Zn}$-ratio). The particle size distribution was obtained from dispersions in MMA. Data reproduced from ref. ${ }^{[58]}$ with the permission of ACS.

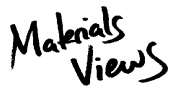

www.MaterialsViews.com
Macromol. Mater. Eng. 2012, 297, 838-863

(c) 2012 WILEY-VCH Verlag GmbH \& Co. KGaA, Weinheim 
concentration of $t \mathrm{BuPO}_{3} \mathrm{H}_{2}$ at full coverage. The systematic decrease of mean diameter and narrowing of the size distribution with increasing $\mathrm{P} / \mathrm{Zn}$ can be considered as a textbook example for particle synthesis with precise control over particle size and surface chemistry by controlled chemical precipitation.

\section{Particles in a Polymerizing Medium}

The addition of pigment powder into a polymerization mixture, namely in situ polymerization, has been recognized as the most widely used strategy to obtain polymer nanocomposites with interesting optical properties. ${ }^{[59]}$ It has been applied to many different polymerization processes such as free-radical chain-growth, ${ }^{[60]}$ stepgrowth, ${ }^{[61]}$ and ring-opening polymerizations. ${ }^{[62]}$ Moreover, a variety of polymerization methods for instance bulk, ${ }^{[63]}$ solution, and multiphase reactions including emulsion, ${ }^{[64]}$ suspension, ${ }^{[65]}$ and dispersion ${ }^{[66]}$ polymerization have been combined with this approach. Bulk polymerization and solution polymerization yield polymer nanocomposites that can be further processed into various shapes either from solution or melt. The multiphase polymerization offers to produce colloidal polymer nanocomposites where the inorganic particles are coated physically or chemically by macromolecules. This type of hybrid particles are a special class of nanocomposites because the polymeric surface layer enables the nanoparticles to be dispersed in desired media (organic or aqueous) depending on surface chemistry. Colloidal nanocomposites carry potential interest in the field of medicine, cosmetics, and printing technologies. ${ }^{[67]}$ Under the heading of "particles in the polymerizing medium," surface-initiated polymerization has to be mentioned as well, in so far as it involves polymerization of monomers radially outward from initiator sites that are immobilized on the particle surface. This approach allows producing inorganic core/ polymer shell type hybrids with a highly dense grafted surface (polymer brushes). Well-defined layers in terms of controllable graft density and uniform length of chains can be precisely produced. ${ }^{[68]}$

Irrespective of polymerization type, nanocomposites prepared by in situ polymerization, in general, exhibit better-defined physical properties compared to the ones prepared by simple mixing (compounding or blending). A striking example supporting this argument is found in the work of Harmon and co-workers ${ }^{[69]}$ PMMA/single-walled carbon nanotube (SWCNT) composites were prepared by in situ polymerization induced by either UV light or ionizing radiation. The composites were dissolved in methylene chloride and then cast into films. Figure 5 presents images of these films. The film of parent PMMA is also shown for comparison. While the sample obtained by melt blending of
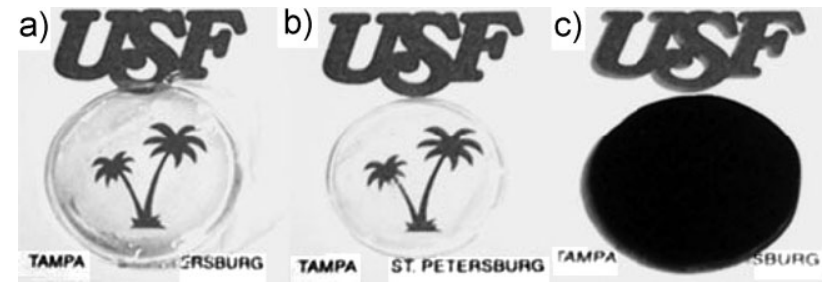

Figure 5. Photographs of discs of different PMMA/SWNT nanocomposites: (a) obtained by in situ UV light induced bulk polymerization of a colloidal dispersion of SWNTs in MMA, (b) neat PMMA for comparison, (c) PMMA/SWNT composite obtained by melt blending. Reprinted from ref. ${ }^{[69]}$ with the permission of Wiley.

premade PMMA and CNT is completely opaque in the visible, the one prepared by in situ polymerization at identical content of nanotubes is as transparent as neat PMMA. The clarity of the film prepared by in situ polymerization is explained by the assumption that individual CNTs are initially well dispersed in the monomer and remain so homogeneously dispersed in the polymer matrix, and is too small to scatter visible light. In the process of melt mixing, the nanoparticles are usually introduced into the melt in powder form, then attempted to be dispersed by extrusion at high temperature. It is highly probable that the particles remain in the polymer matrix entangled as fiber-aggregates, and, therefore, form light scattering fiber-rich domains embedded in the PMMA matrix. The list of examples showing superior physical properties of in situ polymerized nanocomposites compared to those prepared by physical mixing can be extended. In addition to optical clarity exemplified here, dimensional stability after a set of mechanical deformation at high temperatures, ${ }^{[70]}$ flame retardancy, ${ }^{[71]}$ thermal stability, ${ }^{[63 b]}$ and conductivity at low particle content ${ }^{[72]}$ are among the properties that were demonstrated to be improved when the in situ polymerization approach was employed. ${ }^{[73]}$ Such being the case, the question that needs to be answered is for the origin of this behavior. One may argue that it reflects structural differences occurring in the course of preparation. In other words, the presence of particles with large specific surface is suspected to change important details of the mechanism of chain growth in free radical bulk polymerization. There are two cases to be considered. First, one can invoke the interaction of polymer chains as they are formed with the nanoparticle surfaces. This argument refers to the structure of the interface between newly formed macromolecules and the solid nanoparticles, that is, adsorption behavior of polymer chains as they are formed. A premade polymer chain physically mixed with nanoparticles has a minimum of surface contacts since complete adsorption of all chain segments has high entropic cost. In the process of in situ 
polymerization, the nanoparticles are initially wet by the monomer. It may be hypothesized that the surface acts as a template for the chain reaction, thereby altering the mechanism of polymerization and adsorption. The second mechanism that would make in situ polymerization different lies in the reactivity of the particle surface to propagating chain ends. It must be remembered that in the process of in situ polymerization the entire evolution of chains, that is, initiation, growth, and termination of the polymer chains, takes place in a volume confined by the interstices between the solid inorganic particles. Considering the high surface energy and large surface area, the particle surface may interfere with the polymerization process and may alter the microstructure of the polymer as it is formed. This may also result in changes of the architecture of the macromolecules. ${ }^{[64]}$ A further consequence along these lines of thought could be the grafting of chain ends onto the nanoparticle surface. In general, the interaction of polymer and nanoparticles involves non/ covalent interactions; thus, they can be separated from each other via physical means, for example, dissolution of the polymer. In a grafting process, the propagating chain end is coupled to the nanoparticles by a covalent link. Polymer-grafted nanoparticles show structurally different material properties. Most importantly, particles and polymer cannot be separated by physical means. It is, therefore, easy to demonstrate whether such reactions have occurred.

\subsection{Free-Radical Bulk Polymerization in the Presence of Nanoparticles}

This process is schematically depicted in Figure 6. The ex situ prepared and surface-modified pigments are dispersed into the polymerization mixture containing merely the monomer and the nanosized particles. The resulting dispersion is subjected to free-radical polymerization initiated either thermally or by radiation. The mechanism of free-radical polymerization proceeds via generation and propagation of free radicals. The particle surface significantly interacts with the growing macroradicals, and this affects the whole process in terms of kinetics, and microstructure of the resulting polymer. ${ }^{[67]}$ In the following, we will discuss the process in presence of various types of nanoparticles. It must be noted that in the papers reviewed here, the surface of the particles was partly or completely covered with surfactants for the sake of obtaining homogeneous particle dispersion in the bulk monomer, not specifically designed to interfere with radical polymerization. Thus, surface initiated polymerization and any reaction in terms of "grafting from" or "grafting to" approach are excluded.

Carbonaceous nanoparticles (CB and fullerenes including CNT) strongly interfere with free-radical polymerization of vinyl monomers. These particles are composed of polycondensed aromatic rings which make these particles reactive to propagating alkyl radicals. The reactivity of these particles increases as the number of aromatic rings in the structure increases. For instance, the reactivity of CB to methyl radicals is $10^{7}$ times higher compared to that of benzene. ${ }^{[74]}$ Therefore, carbonaceous particles act as radical scavengers independent of morphology of the particles. For example, Ueda et al. ${ }^{[74 \mathrm{~b}]}$ recently showed that the presence of $\mathrm{CB}$ in a polymerization mixture retards free radical polymerization of vinyl monomers such as MMA, vinyl acetate, and styrene. It was reported that the extent of retardation depends on the polarity of the polymerization medium. The effect of retardation decreases in ionic liquids compared to the same polymerization process in toluene. This may be due to the fact that the lifetime of the growing polymer radical is prolonged. CNT and fullerenes have been found relatively more reactive than $\mathrm{CB}$ because the curvature in the spherical particles imparts a significant strain upon the $\mathrm{sp}^{2}$ hybridized carbon bonds. Jia et al. ${ }^{[75]}$ reported similar scavenging effects for CNT for freeradical polymerization of MMA initiated by azoisobutyronitrile (AIBN). The initiator radicals are depleted by the nanotubes, so higher molecular weight polymers are obtained compared to PMMA prepared in absence of the particles. Similarly, $\mathrm{C}_{60}$ fullerene retards polymerization and induction periods were observed in experiments prior to the 
onset of the polymerization. ${ }^{[76]}$ Multiple additions of MMA radicals onto the $\mathrm{C}_{60}$ moiety gives a mixture of compounds differing by the numbers of oligomers bound to the $\mathrm{C}_{60}$ core. Therefore, the in situ polymerization of MMA containing $\mathrm{C}_{60}$ gives high molecular weights and branched and star like structures rather than linear macromolecules. One can also express the results by saying that $C_{60}$ acts as a multifunctional comonomer in free radical polymerization of vinyl compounds.

In fact, this type of reaction has been originally observed in melt compounding of CB with rubber. Due to the high shear rate and high temperature of this process, macromolecules dissociate into radical fragments. The radicals are grafted onto the surface of $\mathrm{CB}$ particles and modify their surface properties. For instance, CB compounded with natural rubber exhibits much enhanced dispersibility in organic media compared to original $\mathrm{CB} .{ }^{[77]}$ Similar phenomena were observed for all carbonaceous particles. Choi et al. reported preparation of PMMA/multi-walled carbon nanotube (MWCNT) nanocomposites by in situ bulk polymerization using AIBN as initiator. ${ }^{[75,78]}$ The interaction between MWCNT and propagating MMA radicals leads to grafting of PMMA chains to the surface of the nanotubes. In a typical in situ "grafting to" process approximately 1020 wt\% of polymer chains become grafted onto the carbonaceous particles depending on the polarity of the medium. ${ }^{[74 b, 79]}$ However, when monomers such as pyrrole ${ }^{[80]}$ and phenylacetylene ${ }^{[81]}$ were polymerized in the presence of CNT, significant chemical attachment of polymer chains onto the tubes was not observed. Rather, there was evidence of physical adsorption of the newly formed chains via non/covalent interactions such that polymer chains precipitated onto the surface of the CNT.

Although the bulk polymerization of a variety of monomers in presence of oxidic and chalcogenidic pigments has been reported for an incredibly large number of systems, the role of these pigments in the polymerization process was not that much attempted to be clarified as is the case for carbonaceous particles. A noteworthy study is the recent paper by Macarie and Ilia ${ }^{[82]}$ who investigated photopolymerization of bisphenol-A epoxydiacrylate - a commercial product for coating purposes - containing various pigments as colorants. These particles were oxides and/or chalcogenides. The particle diameter was below $5 \mu \mathrm{m}$. Surface modification was not attempted and the particle content was fixed to $5 \%$ by weight. Figure $7 \mathrm{a}$ presents conversion versus time curves for the in situ photopolymerization. In comparison with the neat system, the rate of polymerization decreases significantly in the presence of the pigments. The value of the limiting conversion obtained for the monomer/pigment mixture is less than the one for the neat monomer. The data indicate that the particles act as inhibitor and/or retarder in the photo crosslinking process. It must be noted, however that
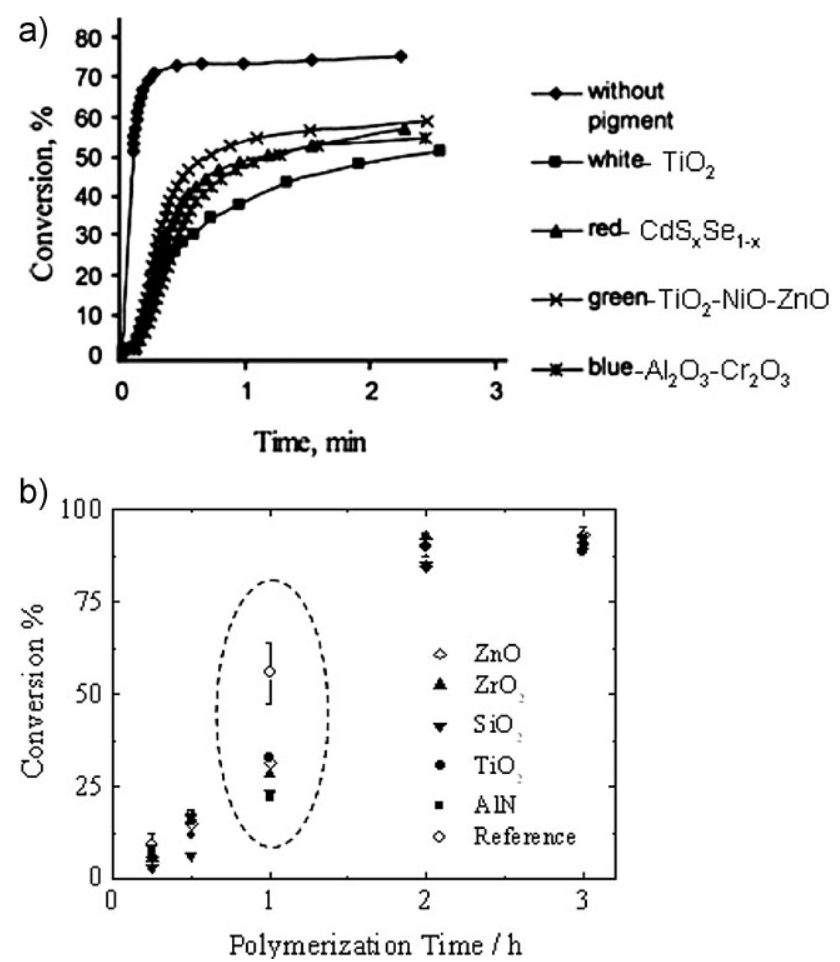

Figure 7. (a) Conversion versus time profiles for UV-induced curing of blends of dimethacrylate with different $\mu \mathrm{m}$ scale pigment particles at identical weight fraction of 0.05 ; The particle size was about $5 \mu \mathrm{m}$. Data reproduced from ref. ${ }^{[82]}$ with permission of Wiley. (b) Conversion of MMA to PMMA at different reaction times in presence or absence of different types of nanoscale inorganic particles as indicated in the Figure at identical weight fraction of loading (0.06); the particle diameters were between 20 and $400 \mathrm{~nm}$. Data reproduced from ref. ${ }^{[63 a]}$ with permission of ACS.

the particle size in these studies was too large to allow the preparation of a homogeneous dispersion. Sedimentation of the pigment will be unavoidable. Therefore, the kinetic features may vary depending on the rate of sedimentation and the method of sampling.

In the light of the foregoing discussions and claims of literature we have studied the effect of various commonly used nanosized particles such as $\mathrm{ZnO}, \mathrm{TiO}_{2}, \mathrm{ZrO}_{2}, \mathrm{SiO}_{2}$, and AlN on the free radical bulk polymerization of MMA. ${ }^{[63]}$ Polymerization was performed in all cases on stable colloidal dispersions. The particles of $\mathrm{ZnO}, \mathrm{TiO}_{2}$, and $\mathrm{ZrO}_{2}$ were surface modified with alkylphosphonic acids to render them dispersible in MMA. AlN and $\mathrm{SiO}_{2}$ were used as obtained without special surface treatment. The pertinent result is that the polymerization in presence of particles strongly deviates from classical behavior. As an example, Figure $7 \mathrm{~b}$ shows conversion versus time profiles for the polymerization in the presence of various types of particles, with their concentration being fixed at 6 wt\%. The 
polymerization behavior of the neat MMA was identical to that known from literature. ${ }^{[83]}$ A sigmoidal increase of conversion with respect to polymerization time was observed in all cases. At intermediate conversions, a steep increase in the rate of conversion takes place. This phenomenon is called autoacceleration, and it has been frequently observed in bulk polymerization of acrylates and methacrylates. It is commonly explained invoking that the termination is a diffusion-controlled process. As the monomer is converted to polymer, the viscosity of the reaction medium increases. The diffusion of the macroradicals and, therefore termination becomes hampered. In consequence, the rate of termination rapidly slows down, while the rate of propagation remains unaffected. The concentration of radical increases as termination is suppressed, and thus autoacceleration in the polymerization comes unavoidably into play. The name "gel effect" has been popularized in the description of this sequence of events. When nanosized particles are present, they seem to reduce the rate of polymerization. Even so complete conversion can be achieved as for the neat monomer at roughly the same reaction time. It seems that the particles act as neither inhibitor nor retarder, instead, they prevent formation of the undesirable gel effect which causes systematic development of bimodal molecular weight distributions in free radical polymerization and, moreover, the distribution gets broader on the high molecular weight side. The particles suppress the formation of high $\bar{M}_{\mathrm{w}}$ chains, at least their appearance is delayed to the very late stages of polymerization. ${ }^{[63 \mathrm{~b}]}$ Figure 8 shows MWD of in situ formed polymer which was retrieved at the point where the gel effect regime began. The presence of the particles suppresses the formation of a high $-\bar{M}_{\mathrm{w}}$ fraction in the distribution almost completely regardless of the type of particles. While our results are confined to spherical nanoparticles, two-dimensional layered particles seem to show similar, if not identical effects. Achilias et al. ${ }^{[84]}$ recently reported the effect of montmorillonite and organically modified montmorillonite (Cloisite) on free radical polymerization of MMA initiated by dibenzoyl peroxide (BPO). Conversion versus time and molecular weight distribution were strongly affected by the presence of the clay nanoparticles particularly in the regime of the gel effect in the same way as expected form our results.

In contrast to carbonaceous particles, in situ grafting of chains to the particle surface has not been observed in bulk free-radical polymerization when metal-oxide-based nanoparticles were present. (An exception are special surface treatments such as radiolysis. ${ }^{[85]}$ In the examples given in Figure $7 \mathrm{~b}$ and $8, \mathrm{ZnO}$ and $\mathrm{ZrO}_{2}$ particles were quantitatively retrieved from PMMA by dissolution and redispersed into fresh MMA. The size of the particles was compared using DLS before and after polymerization. Identical size and size distribution was obtained proving that grafting to their surface had not taken place. This is shown for representative cases in Figure 9.

\subsection{Chain Termination}

In general, a propagating macroradical is terminated through either combination or disproportionation. In the former, two propagating macroradicals couple to each other and a head-to-head linkage appears in the resulting macromolecule. This linkage appears as one of the "weak linkages" in the polymer backbone. In the latter case, two chains of the size of the macroradicals, however with different terminal groups are formed: one is saturated and the other one exhibits a terminal double bond. This end group enhances thermal degradation of polymer chains in particular cases when unzipping of monomer residues from

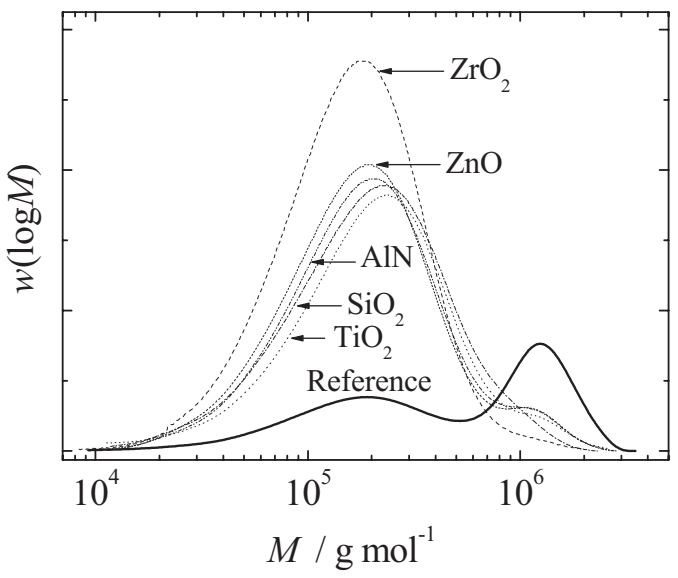

Figure 8. Molecular weight distributions of PMMAs recovered after 30 min polymerization time as indicated in Figure $7 \mathrm{~b}$. Data reprinted from ref. ${ }^{\left[{ }_{3} a\right]}$ with permission of ACS.
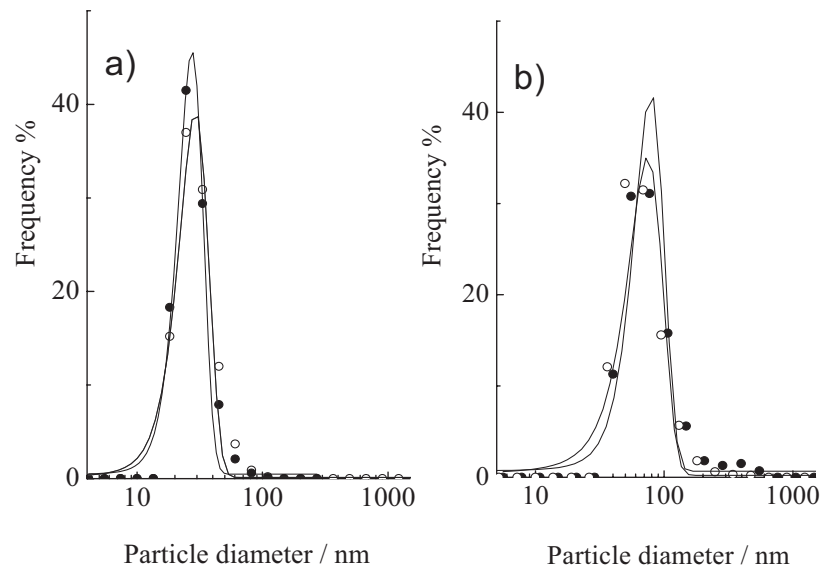

Figure 9. Size distribution of nanoscale particles before (open points) and after polymerization (filled points) for (a) $\mathrm{ZnO}$ and (b) $\mathrm{ZrO}_{2}$. Data reproduced from ref. ${ }^{[63 \mathrm{a}]}$ with permission of ACS.

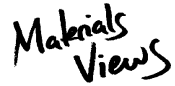

www.MaterialsViews.com 
the terminal sites is available as a mechanism of degradation. ${ }^{[86]}$

PMMA obtained by radical polymerization degrades thermally by three steps: (i) mass loss at $188^{\circ} \mathrm{C}$ originating from head-to-head linkages formed by combination, (ii) mass loss at $300^{\circ} \mathrm{C}$ originating from vinylidene ends formed by disproportionation, and (iii) mass loss due to random scission of the polymer backbone. Thus, the inspection of thermal degradation allows for identification of chain defects as consequence of their history of formation. For example, in anionic polymerization the formation of abnormal linkages, that is, head-to-head linkage and vinylidene end groups, is unlikely. The polymer is merely composed of head-to-tail linkages, that is, free of defects. Therefore, anionically synthesized PMMA undergoes degradation in a single step by random scission, and thus shows higher thermal stability compared to PMMA obtained by bulk polymerization of neat MMA. ${ }^{\text {[86] }}$

The mechanism of how propagating radicals are terminated depends on the chemistry of the particles present. The termination occurs by surface grafting with carbonaceous particles. ${ }^{[78,87]}$ This was confirmed for all carbon allotropes. ${ }^{[76,78]}$ On the other hand, oxide- and nitride-based particles support a degenerative transfer reaction between the particle surface and the propagating free radical. ${ }^{[63]}$ The surface of metal oxides and nitrides is presumably covered with a few layers of chemisorbed water ("non-freezing" water) which may be reactive in terms of transfer reactions. This transfer reaction suppresses the autoacceleration by keeping the radical concentration low enough because of slow reinitiation.

The occurrence of this degenerative transfer is supported by recent findings of Anzlovar et al. ${ }^{[88]}$ who analyzed the chain growth of PMMA in situ at different $\mathrm{ZnO}$ content by ${ }^{1}$ H NMR spectroscopy. The signal of vinylidene protons that are end groups, normalized with respect to methyl protons of the backbone was followed. The intensity of this signal was found to diminish as the particle fraction increased, and this is direct evidence for the degenerative action as was proposed. The same authors also demonstrated that the particle size is an important parameter. While the reduction of termination reaction by disproportionation was clearly observed for nanosized particles of $75 \mathrm{~nm}$, only small changes were observed for larger particles $(0.34 \mu \mathrm{m})$. This result is not surprising since the probability of a radical chain to encounter a particle surface during lifetime must be proportional to the specific surface area of the particles; hence, the smaller the particles size the larger the effects to be seen at constant loading of the system with the solid phase.

The proposed degenerative transfer will not induce nor control the livingness of the polymerization contrary to what is known for atom-transfer radical polymerization (ATRP). Various surface functional groups of nanoparticles may take part in this transfer reaction. However, as common species that are already present on the surface of the nanoparticles, high density of surface bound water and surface-adsorbed oxygen are the first species one would speculate to be involved. A further systematic study, for example, by electron paramagnetic resonance (EPR) spectroscopy would be interesting to understand the process of in situ polymerizations in presence of nanosized particles better.

As outlined above, the presence of nanosized particles suppresses the formation of thermally unstable vinylidene end groups in the polymerization of MMA. Therefore, a thermally more stable polymer, that is, nanocomposite material, is obtained. A clear evidence is shown in Figure 10 where the degradation profiles of differently prepared PMMA/ZnO nanocomposites are displayed. Blending of ZnO nanoparticles with preformed PMMA does not lead to an improvement in thermal stability compared to the neat polymer. The mass loss versus temperature diagram shows the fingerprint due to the weak links and end groups present as a consequence of termination. The degradation of PMMA/ZnO nanocomposites prepared through in situ polymerization is shifted to the random scission regime, namely to higher temperatures. PMMA prepared in the presence of various other oxide and nitride nanoparticles exhibits similar superior thermal stability in comparison to both neat PMMA ${ }^{[63 a, 84 b, 89]}$ and the nanocomposites prepared by mere blending. ${ }^{[63 \mathrm{~b}]}$

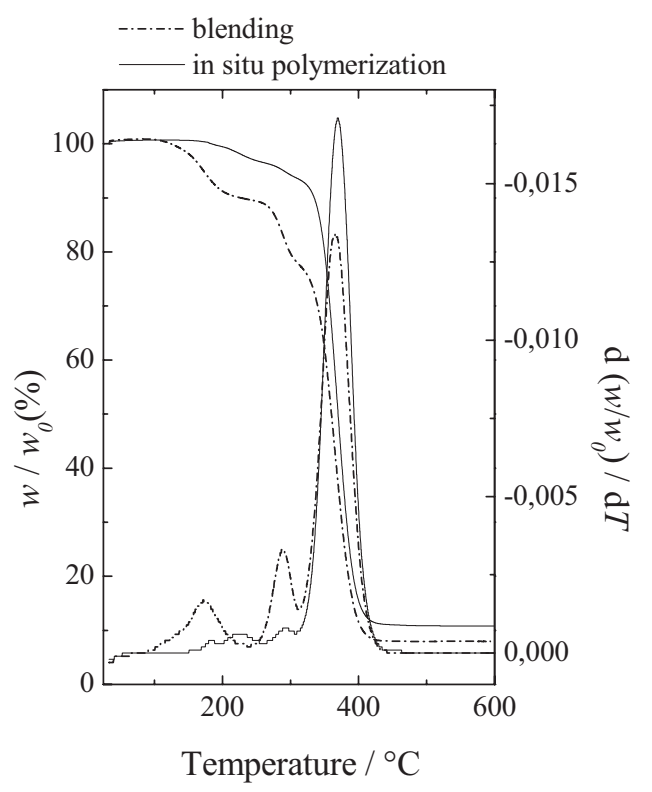

Figure 10. Mass loss and differential mass loss of PMMA/ZnO nanocomposites as obtained by in situ bulk polymerization of MMA (full line) in comparison to neat PMMA (bulk polymerized broken line); or a physical blend of particles and neat PMMA. Data reproduced from ref. ${ }^{[63 b]}$ with permission of Wiley. 


\section{Particle/Polymer Interaction}

\subsection{Depletion Attraction}

In the process of in situ polymerization, monomer/ nanoparticle dispersion is gradually turned into a bulk polymer/nanoparticle composite. The emergence of nonadsorbing polymer in the dispersion results in an effective interparticle attraction via the "depletion" mechanism. ${ }^{[90]}$ (Figure 11a) Even though the particles are dispersed individually in the monomer, they undergo clustering in the resulting polymer matrix. This feature can be explained as follows. Macromolecules maintain a configuration which is energetically the most favorable one, that is, they form a random coil. In the presence of particles, the chains cannot approach to a particle surface closer than a certain distance because the coil would have to adopt locally an extended conformation which is in fact less favorable due to loss of conformational entropy. Thus, a polymer-free depletion zone exists around each particle. At low particle content, the interparticle distance is larger than the depletion zone. As the concentration of particles increases, the depletion zones begin to overlap. The chains present in between the particles escape. In turn this produces a local osmotic pressure, that is, offset by interparticle forces. ${ }^{[91]}$ In the end, particle-rich domains (clusters) are developed in the bulk polymer matrix. ${ }^{[92]}$ Figure $11 \mathrm{~b}$ shows a representative electron micrograph of a polymer/particle composite showing very clear evidence for the depletion attraction phenomenon. ZnO nanoparticles of $22 \mathrm{~nm}$ diameter were initially dispersed in MMA homogeneously. Upon polymerization, ZnO domains are formed which consists of loosely packed ZnO nanoparticles. These domains are evenly dispersed in the bulk as separate islands (particle clouds) ${ }^{[63]}$ In between the particle domains, polymer-rich domains (devoid of particles) are identified. The process leading to such heterogeneous particle distributions is also called depletion flocculation, a well-known phenomenon in colloid science. ${ }^{[93]}$

\subsection{Prevention/Suppression of Depletion Flocculation by Crosslinking}

An approach that has gained considerable popularity to overcome undesirable particle aggregation due to the depletion flocculation is to create in situ a network structure in the polymerization medium. Since the particles are not aggregated in the monomer, crosslinking prevents the desired diffusion of the particles during polymerization. If the rate of crosslinking is faster than the diffusion rate of the particles, individual particles stay confined in meshes of the polymer network knitted by branching/crosslinking centers. Thereby, the separation of initially well-dispersed particles becomes kinetically hindered. A homogeneous dispersion of particles is eventually achieved and the nanoparticles stay localized, which may be very important for the function of the material. This strategy is highly analogous to vulcanization in the rubber industry where it is necessary to achieve a highly homogeneous distribution of $\mathrm{CB}$ particles within the crosslinked rubber matrix. A three-dimensional network can be generated in polymer/ particle nanocomposites by linking of in situ formed chains either with each other or with the particle surface. Figure 12 schematically shows two different approaches. In the first approach, a polymerizable surfactant is used. Surfaceanchored groups of the particles participate in the polymerization such that the in situ formed polymer chains are covalently grafted to the particle surface. Thereby, the migration of the particles and in consequence depletion flocculation is prevented. Another strategy to create a three-dimensional network in the course of the polymerization is to introduce a multifunctional comonomer. The newly formed polymer chains become mutually linked via covalent bonds, and the particles become confined in the meshes of the polymer network as it is formed.

\subsubsection{Polymerizable Surfactants}

A polymerizable surfactant is a molecule composed of two functional groups. One of them is meant to anchor the molecule on the particle surface. Another group is designed to act as comonomer in the polymerization. This dual function leads to the formation of stable covalent links between polymer and particles in the vicinity of the particle surface. In fact, many functional groups may interact
Figure 11. Left: The effect of depletion attraction among particles, reprinted from ref. ${ }^{\text {[93] }}$ with permission of IOP. Right: TEM micrograph of a representative polymer/nanoparticle (PMMA/ZnO) composite showing interparticle depletion attraction. Data repinted from ref. ${ }^{\left[6_{3} b\right]}$ with the permission of Wiley.
Macromol. Mater. Eng. 2012, 297, 838-863 (c) 2012 WILEY-VCH Verlag GmbH \& Co. KGaA, Weinheim 


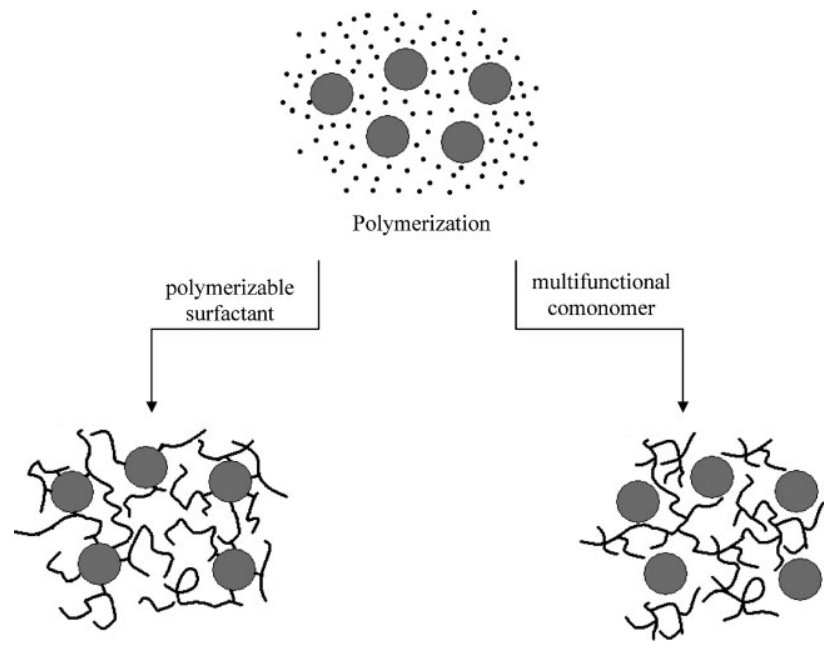

Figure 12. Suppression of depletion attraction ("depletion flocculation") by bulk polymerization of monomer/particle blends in presence of either polymerizable surfactants that act as crosslinkers or multifunctional monomers which yield highly branched networks and thereby encage the particles.

with the polymer chains on the same nanoparticle. Moreover, one long polymer chain may tether more than one particle. Consequently, network structures are obtained in which the particles act as multifunctional crosslinks that are immobilized in a permanent network.

Schurtenberger et al. showed a clear proof that nanoparticles can be prevented to cluster using a polymerizable surfactant. Their nanocomposite system was based on silica particles with a radius of $25 \mathrm{~nm}$, modified by 3-(trimethoxysilyl)propyl methacrylate, a polymerizable surfactant. The polymerization was induced by UV light with a suitable initiator added. The particle dynamics in the process of in situ bulk polymerization was quantitatively monitored by time resolved scattering methods. ${ }^{[94]}$ Two non-invasive methods were combined. The Brownian motion of the silica particles was monitored by DLS. The evolution of the static structure and the possible formation of particle agglomeration were investigated by time-resolved small-angle neutron scattering (SANS). The particles initially showed Brownian diffusion. As the PMMA chains started to grow in the bulk monomer they became as well chemically attached to the particle surface. Thus, the silica NPs were localized by surface bound PMMA chains and no longer underwent Brownian motion. This change in particle dynamics was accompanied by a tremendous increase of the viscosity of the sample as consequence of crosslinking and a subsequent transition to a solid-like state took place. Gradual reduction in the particle diffusion rate as the polymerization proceeded was identified by the shift of the correlation time to longer decays. Figure 13a presents the time evolution parameter $p$ from analysis of the mean square displacement of the particles calculated from the autocorrelation function with respect to polymerization time. $p$ shows a remarkable decrease within the polymerization time. Figure $13 \mathrm{~b}$ shows the scattering profiles of a typical reaction mixture at different times of polymerization. The profiles give almost identical signals over a wide range of scattering vector suggesting that a measurable aggregation does not take place in the course the bulk polymerization. The silica particles remain non-aggregated in PMMA while the surface of silica particles provides crosslinks. The results of these investigations clearly indicate that in situ crosslinking is a suitable way to achieve homogeneous dispersion of nanoparticles in a bulk polymer medium.

The effect of crosslinks generated by polymerizable surfactants on particle dispersions can also be seen in a)

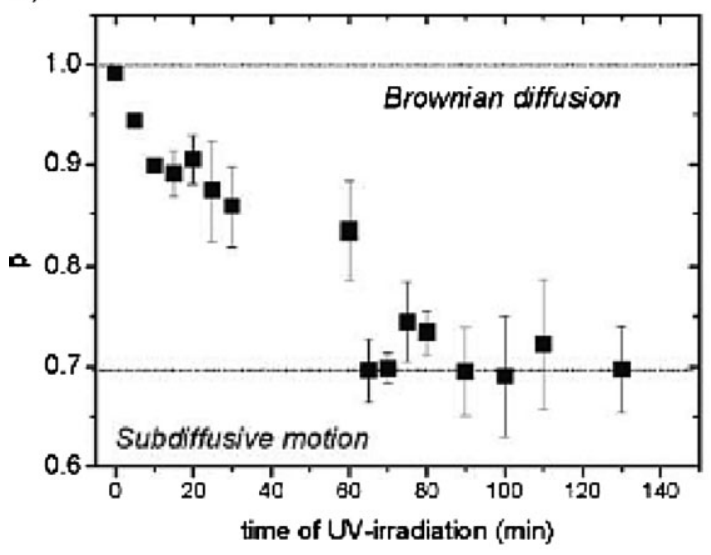

b)

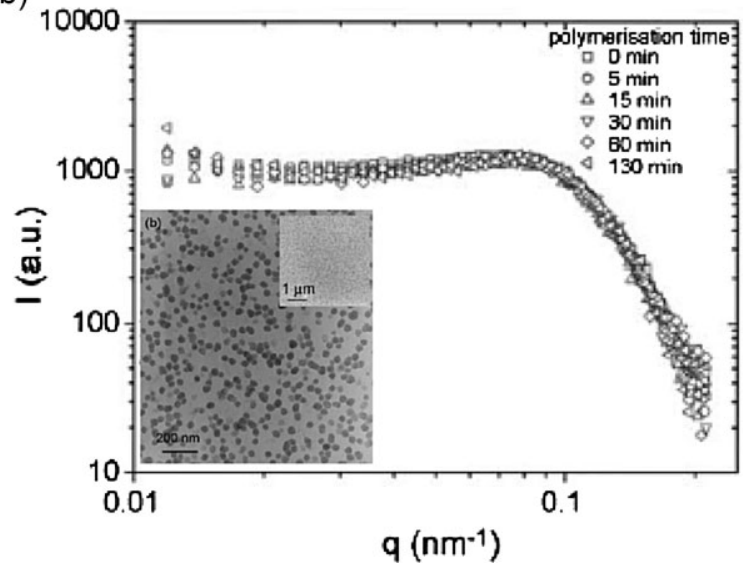

Figure 13. (a) Time evolution parameter $p$ during radical polymerization of MMA in presence of surface modified silica. (b) Time-revolved SANS for a sample containing 13 wt\% percent silica; the inset shows the respective TEM micrographs. All data reproduced form ref. ${ }^{\left[{ }^{[9]} \text { with }\right.}$ permission of Elsevier Publishers. 
the work of Hung and Whang. ${ }^{[57]}$ In this particular nanocomposite, methacryloxypropyltrimethoxysilane (MPS)-modified ZnO nanoparticles were obtained by controlled chemical precipitation. The surface modification was performed in situ during particle growth and poly(2-hydroxyethyl methacrylate) (PHEMA)-based nanocomposite was subsequently prepared by in situ bulk polymerization. The level of dispersion of both surface-modified and neat $\mathrm{ZnO}$ particles was compared. While large particle domains were observed in the nanocomposites prepared from the neat particles, the modified particles were well dispersed and non-aggregated in the PHEMA matrix. This approach has been successfully applied for various particle/ polymer systems. Yang and co-workers ${ }^{[37]}$ demonstrated the preparation of transparent $\mathrm{CdTe} /$ polystyrene (PS)/ PMMA bulk nanocomposites from water-dispersible nanocrystals using polymerizable OVDAC. Surfactants of similar structure but without copolymerizable function did not work but resulted in opaque nanocomposites due to the strong flocculation of the nanoparticles. In another example, Sugimoto et al. ${ }^{[95]}$ reported the preparation of transparent and thermally stable PMMA nanocomposites including reactive silica NPs prepared with the polymerizable surfactant 2-methacryloxyethyl isocyanate for in situ bulk polymerization. The isocyanate groups presumably interact with the hydroxyl groups on the silica surface. The modified silica particles were copolymerized with MMA. The authors claimed that the resulting hybrid materials exhibit excellent transparency, thermal stability, high elastic modulus, and good surface hardness. The enhancement in the physical properties was attributed to the formation of chemical links between the particulate inorganic phases and the surrounding bulk polymer.

\subsubsection{Multifunctional Comonomers}

The introduction of trace amounts of a multifunctional comonomer to the reaction medium causes branching and/ or crosslinking. This will also help to efficiently suppress flocculation of the nanoparticles. Our group has taken this approach using the bifunctional ethylene dimethacrylate (EDMA) as comonomer for the in situ polymerizaton of stable ZnO/MMA dispersions. ${ }^{[63 a]}$ Figure 14 presents two PMMA/ZnO nanocomposites that have the same amount of identical ZnO nanoparticles. The weight fraction of ZnO particles was 0.05 in both cases. One of the nanocomposites (Figure 14a) is made up of only MMA and initiator; and the resulting chains have a linear architecture. The mechanism of depletion flocculation comes into play and clustering occurs. However, the nanocomposite prepared in presence of a trace amount of a difunctional comonomer contains crosslinked and/or branched PMMA chains. The micrograph of this nanocomposite shows mainly individual particles and no indication of flocculation. a)

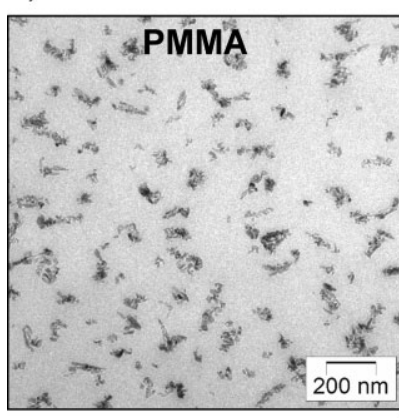

b)

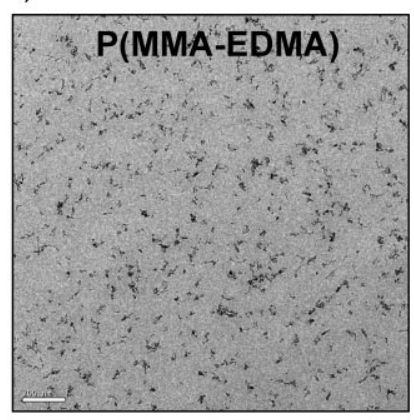

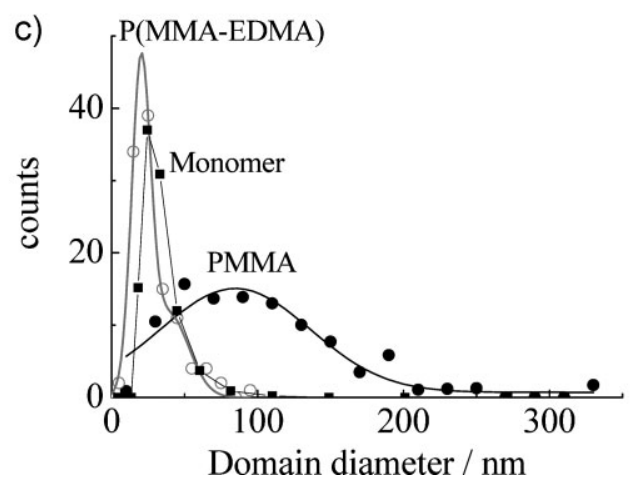

Figure 14. TEM micrographs of PMMA/ZnO nanocomposites prepared by in situ bulk polymerization of MMA (a) and bulk copolymerization of MMA with EDMA (b). The size distribution of the domains obtained for either (a) or (b) are shown in (c). Data reproduced from ref. ${ }^{\left[{ }^{63 a}\right]}$ by permission of ACS.

Using a difunctional comonomer is sufficient to prepare a random network structure but does not offer precise control over the homogeneity of the crosslink density. An ideal polymer network with uniform chain length between junction points was prepared by reaction of hydroxylterminated polydimethyl siloxane chains and tetraorthosilane (TEOS). ${ }^{[96]}$ This polycondensation was carried out in the presence of fumed silica particles with a diameter of $14 \mathrm{~nm}$. The surface of these particles presents $\mathrm{OH}$ groups which also take part in the condensation process. Thus, the particle surface becomes covalently linked to the surrounding polymer chains. The network thus obtained swelled extensively, if exposed to solvents like toluene or benzene. However, the silica particles could not be extracted from the gels or swollen gels. In conclusion, the particles are essentially trapped in the network and this guarantees a long lasting homogeneous distribution of individual particles in the bulk polymer.

\subsubsection{Simulation and Model}

According to experimental results mentioned above, a better and stable dispersion of nanoparticles in a polymer matrix is achieved when the polymer is branched or crosslinked. Recently, Zhao et al. ${ }^{[97]}$ validated this strategy

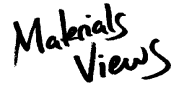

www.MaterialsViews.com 


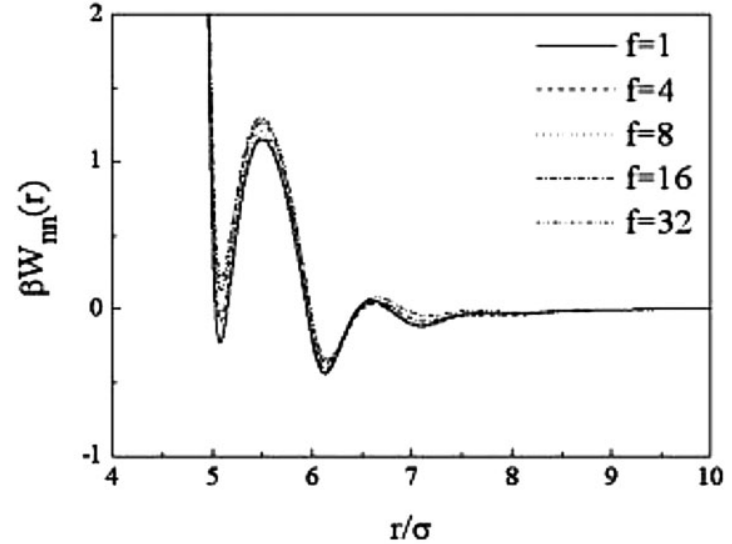

Figure 15. Interaction potential in presence of star polymers with different number of branches; reproduced from ref. ${ }^{[97]}$ by permission of ACS.

employing a polymer reference interaction site model (PRISM)-based theory for star polymers. The junction connecting the arms in a star polymer is topologically analogous to the crosslink in a network. ${ }^{[98]}$ Several chains emanate from the junction in the star much alike the arms in network, the difference being that the emanating chains have dangling ends in the star, but are ties to the next crosslink in the network. The authors studied the structure and effective interactions in star-polymer/nanocomposite melts focusing on the regime where the radius of the nanoparticle is smaller than radius of gyration $\left(R_{\mathrm{g}}\right)$ of the star polymer. Figure 15 shows the mean force potential for different arm numbers at fixed nanoparticle/monomer strengths. $W(r)$ is the potential of mean force, $\beta$ is $1 / k_{\mathrm{B}} T, f$ refers to the arm number, and $\sigma$ is the monomer size. Evidently, there exist three minima showing negative attractive potential. As the arm number increases, the minimum values at $r / \sigma \approx 5.0,6.0$, and 7.0 decreases indicating that the increase of arm number can well suppress the direct contacts and bridging structures for moderate nanoparticle-monomer attraction in star polymer melts. This indicates that star polymers are excellent dispersant for nanoparticles.

\section{Optical Properties of Nanocomposites}

\subsection{General Consideration}

Interaction of light with a solid includes reflection, refraction, absorption, and scattering of the incident light. Figure 16 presents schematically the interaction of a beam of unpolarized light with an idealized composite film. Let us consider a model system composed of nanosized particle domains homogeneously dispersed into a transparent medium. When a beam of light strikes on such a material,

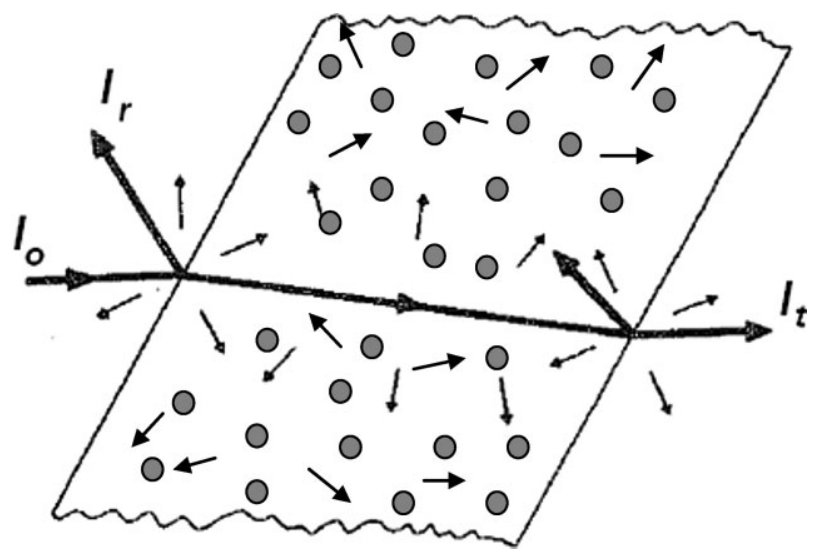

Figure 16. Interaction of a beam of light with a polymer/particle composite; multiple scattering events are indicated. The scheme is adopted from ref. ${ }^{\text {[99] }}$

it is partially reflected from the surface and partially transmitted into the material. If the surface is perfectly smooth, the ray is reflected back from the surface such that the angle of incidence equals the angle of reflection. Reflection from a smooth surface is called specular reflection. If the surface is not smooth but rough, it reflects light into various directions. This is called surface scattering, also known as diffuse reflection. The visual appearance of a bulk material is determined by the relative proportion of these two processes. If the reflected light predominates, the surface looks glossy, but if the surface-scattered light is the major component, it will be matt.

The fraction of incident light which is transmitted into the material, that is, the refracted beam of light encounters the internal microstructure of the material. The intensity of the beam decreases as it travels inside the material $\left(I_{t}<I_{0}\right)$. The reduction in intensity is called attenuation. Two different processes contribute to the attenuation. The first one is absorption, by which the energy of light is converted into other forms of energy such as heat. The second one is scattering in which the energy of light is not changed but spread in all directions away from the path of the beam. Potential scatterers in a bulk polymer are density fluctuations, voids, and/or particulate additives. The attenuation of light occurs due to the combined action of absorption and scattering. The ability of a specimen to transmit incident light is defined as transparency. ${ }^{[99]}$ More technically, it refers to the ratio of intensities of the transmitted to the incident ray. The obvious limiting behavior is encountered when an incident beam of light is transmitted without being scattered or deflected, and fine details of an object situated behind the material (e.g., a film) are perfectly visible. The other limit is opaqueness, where no light is directly transmitted and, objects situated behind cannot be optically resolved. The material itself appears milky. Translucency lies in between these two extremes. The 
sample still transmits the light; however, fine details of an object situated behind are blurred and not discernible. Generally speaking, objects made of polymers fall into the last category. Haze is a routinely used term to describe their optical properties. Haze is defined as the fraction of transmitted light that deviates from the directly transmitted ray more than $2.5^{\circ} .^{\text {[99] }}$ The deflection of the light results in a deterioration of the image of an object, that is, placed behind a hazy piece of material. The hazier a sample is, the less is its transparency. There are obvious connections between haze and transparency. Both are dimensionless quantities and describe aspects of the degradation of image quality by scattered light. Unlike transparency, haze is thickness-independent.

Since the RI of inorganics is higher, in general, than of organics, particles of inorganics act as strong scatterers, and, therefore, the transparency of a polymer/nanoparticle composite system is directly related to its scattering power. The loss of intensity due to scattering can be estimated from Rayleigh's formula:

$$
T=\frac{I}{I_{0}}=\exp \left\{-\frac{32 \pi^{4} \phi_{\mathrm{p}} x r^{3} n_{\mathrm{m}}^{4}}{\lambda^{4}}\left[\frac{\left(n_{\mathrm{p}} / n_{\mathrm{m}}\right)^{2}-1}{\left(n_{\mathrm{p}} / n_{\mathrm{m}}\right)^{2}+2}\right]^{2}\right\}
$$

where $I$ and $I_{0}$ are the intensities of the transmitted and incident light, respectively, $\lambda$ the wavelength of light, $x$ the thickness of the film, $\phi$ the volume fraction of inorganic phase, $r$ the radius of the scatterers assumed to be spherical, and $n_{\mathrm{p}}$ and $n_{\mathrm{m}}$ are the RIs of the particles and the matrix, respectively. This expression was formulated for small (small compared to the wave length of light), nonabsorbing, and isotropically scattering particles in the path of a beam of non-polarized light. However, two uncertainties appear in this formula if applied for polymer/ nanoparticle composite systems. The first one is flocculation. As flocculation takes place, domains of particles are formed which now act as scatterers. Moreover, as the particle content increases, the domains get larger and grow beyond the size $(r<\lambda / 20)$ for which Equation 1 is valid. The second problem in attempts to make use of Equation 1 is the occurrence of multiple scattering, which is completely neglected. When the incident radiation is scattered by only one localized center within a given volume, this event is called single scattering. However, the incident light may be scattered many times within the volume through which it is traveling before leaving the system. This is known as multiple scattering. (cf. Figure 16) Multiple scattering has two consequences. Firstly, the pathlength of light within the specimen is longer than accounted for by the specimen dimensions. Therefore, the intensity of the light diminishes more rapidly. Secondly, and more importantly, it is no longer possible to predict the directional distribution of the scattered light emerging from the specimen, and in consequence an image behind the specimen becomes blurred. Multiple scattering effects become particularly significant for thick samples where the scattering volume is large and the number of scatterers per unit path length is high. This explains the common experience as the thickness of any transparent material is increased, its appearance changes from transparent to translucent and then to opaque. This is probably why in the majority of the recent research papers where transparency of nanocomposites is claimed, it is solely examined on very thin films (thereby, restricting the scattering volume and path length) and at low particle concentration. Under these circumstances, transparency becomes guaranteed albeit far from regimes of real application.

The loss of incident light by scattering can be monitored in two different ways that are schematically shown in Figure 17. The most popular way to characterize transparency is by means of measurement of the amount of light transmitted through a thin film (specular transmittance). The films are usually prepared by spin-coating and their thickness lies in the range of $1-2 \mu \mathrm{m}$. In this method (Figure 17a), the light impinges at normal incidences on the film placed on a transparent substrate. As the light path is blocked by particle-rich domains it is only allowed to pass through polymer-depleted domains. The area occupied by the particles and their clouds is accounted for by their geometrical cross-section. However, the interaction volume

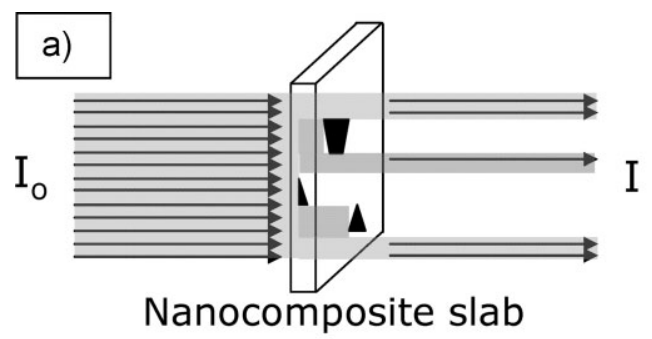

b)

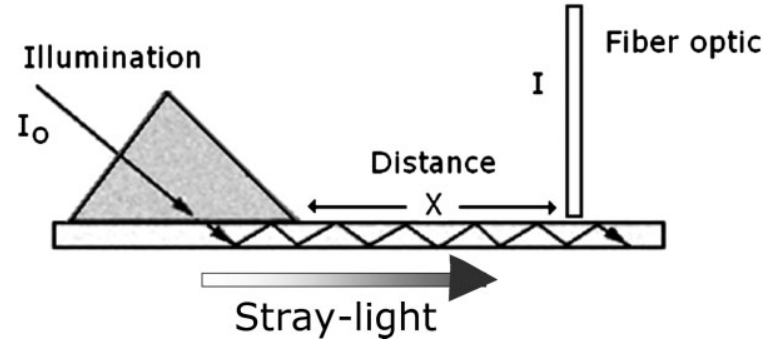

Figure 17. Characterization of transmission of polymer/particle composites: (a) transmission of a thin film at normal incidence of the probe beam and $(b)$ measuring the attenuation of light using a thin film as waveguide and measuring the intensity of stray light.

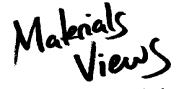

www.MaterialsViews.com
Macromol. Mater. Eng. 2012, 297, 838-863

(c) 2012 WILEY-VCH Verlag GmbH \& Co. KGaA, Weinheim 
of the incident beam is limited by its short path length in the film. While this setup was in fact originally designed for the measurement of absorption; only, it is commonly used for measurement of transmission of polymer nanocomposites as well because its implementation is seemingly easy and practical. As pointed out before, the restriction in scattering volume, that is, the short light path within the film of only a few $\mu \mathrm{ms}$ will not give a realistic picture of the materials properties. Another more precise but less frequent approach to determine transparency is to make use of waveguiding. It is based on the measurement of the intensity of stray light emitted from a probe beam, that is, guided in a film. In a typical setup a beam of monochromatic laser light is coupled via a prism into a film of the composite. This film serves as waveguide by internal reflection of the beam from the surfaces of the film. The beam is confined to the film. Interaction of the beam with particles results in scattering of light in all directions. Most of the scattered light will pass through the film surfaces because it does not fit to the waveguide conditions. As indicated in Figure 17b one can pick up the intensity of stray light as a function of the distance from the point where the probe beam has entered the waveguide. This is practically achieved moving a light guiding fiber, coupled to a CCD camera along the film. This method accounts for the loss of light due to scattering over long path lengths. For comparison, transmission spectra and waveguide loss data of films of neat PMMA and PMMA/ZnO composites are presented in Figure 18. The films of identical thickness were prepared by spin-coating. The transmission spectra (Panel a), demonstrate the high absorption of the composite film in the UV region due to the presence of ZnO that has a high absorption coefficient in this region of the spectrum. ${ }^{[100]}$ Based on these transmission data of the nanocomposite film over the visible region,
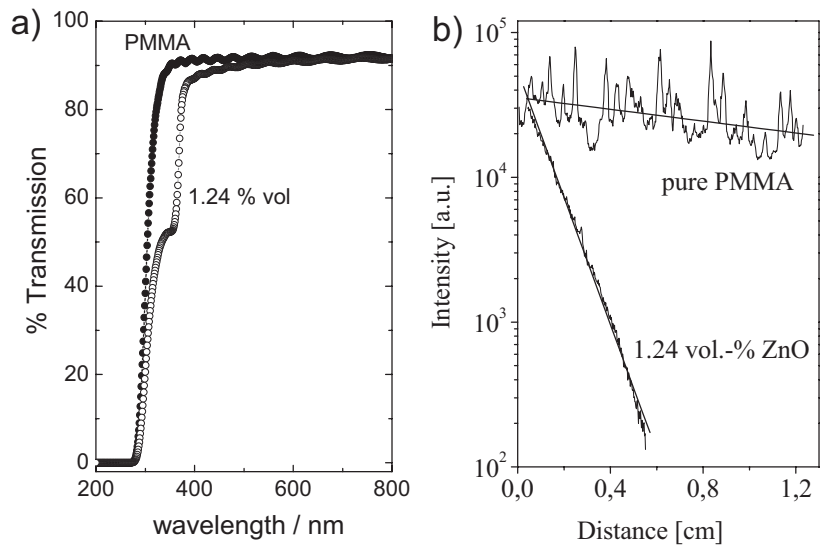

Figure 18. (a) Transmission spectra of a thin film $(d=2.4 \mu \mathrm{m})$ of neat PMMA and of PMMA containing 1.24 vol\% of $\mathrm{ZnO}$ particles. (b) Attenuation of light intensity of the same films for light of $633 \mathrm{~nm}$ wave length in the waveguide configuration (see Figure $17 \mathrm{~b}$ ); reprinted from ref. ${ }^{\left[{ }^{88]}\right.}$ with permission of ACS. the PMMA/ZnO film seems almost as transparent as neat PMMA and the loss by scattering of the incident beam is not readily discerned even if the particle content is $11 \mathrm{wt} \%$ ( $\approx 1.24$ vol\%). The waveguide experiment reveals another picture. Panel $b$ shows the attenuation of the propagating beam by stray light over the path length. Unlike in neat PMMA the intensity of the light beam drops by several orders of magnitude if it propagates in the composite films over short distances. The PMMA/ZnO composite film, which appeared as transparent in the transmission experiment, exhibits its strong scattering power under conditions of the waveguide setup. While the fluctuations of stray light intensity in neat PMMA is mainly due to extrinsic losses caused by impurities such as dust, this fluctuation is barely observed for the composite films because the strong scattering by the ZnO particles overrules these effects completely.

The RI is an essential feature that must be controlled in optical materials. It is an intrinsic property defined as a ratio of the velocity of light in vacuum relative to that in the medium under consideration. It depends on the density and polarizibility of the material. Polymers typically exhibit refractive indices between 1.4 and $1.7 \cdot{ }^{[101]}$ On the other hand, most inorganics have a higher RI in the range of 2.05.0. ${ }^{[5]}$ The blending of the two dissimilar materials into a composite structure seemingly offers routes to design and optimize new composite materials in terms of RI with tunable optical properties. Based on reports in the literature, it seems to be established that the RI of composite materials can be tuned by the particle concentration. ${ }^{[102]}$ However, the RI of composite materials does not obey simple mixing rules. Attempts to estimate the RI of a composite rely on various empirical and semi-empirical models. ${ }^{[103]}$ Effective medium models based on the Maxwell-Garnett theory have been widely used in which monodispersed spheres are arranged in a cubic lattice within a continuous matrix of background RI. The RI of composite material is assumed to be additive based on the volume fraction of the components. However, substantial deviations of the experimental results (both negative ${ }^{[58,104]}$ and positive ${ }^{[103]}$ ) from the simple model were observed for various polymer/nanoparticle systems. Negative deviations can be explained for two main reasons: The first one considers the interfacial volume of the particles coated with surfactant. Each particle is surrounded by a surfactant layer, which is in fact a key requirement to reach compatibility between the inorganic particles and the polymers. Although the surfactant layer is very thin compared to the particle dimensions, the contribution of this layer to the overall volume of the particles may be quite large. The surfactant shell should exhibit a lower RI than the core itself. The second source of negative deviations could be found in quantum confinement effects. When the size of materials, particularly semiconducting materials, is 
reduced to the nanometer scale, the RI is found to be lower than that of the corresponding bulk materials. ${ }^{[42]}$ On the other hand, the main source of positive deviation could be the interaction of the inorganic moiety with the host polymer at the molecular level. As a recent example, Rao and $C h e n^{[103]}$ demonstrated a molecular level dispersion of $\mathrm{TiO}_{2}$ in a polystyrene-based matrix using sol/gel chemistry. A detailed examination by microscopy, allowed to conclude absence of aggregation of the particles. These authors further claimed that some kind of bonding between the inorganic species and the polymer significantly changes both the polarizibility as well as the local density of the materials. Therefore, the contribution of the inorganic species is larger than predicted by an effective medium treatment on account of only two components present in the composite. They emphasize that the RI of such composites cannot be adequately described using an effective medium theory. The same group of authors also presented a new emprical equation to describe the RI of composites, namely

$$
n_{\mathrm{c}}=n_{\mathrm{p}}-\Delta n V_{\mathrm{p}}^{2}
$$

where $n_{c}$ is the RI of the composite, $n_{p}$ the RI of the particles, $\Delta n$ RI the difference in RI between the polymer and particles, and $V_{\mathrm{p}}$ is the volume fraction of polymer. The equation is based on subtraction of the polymer contribution from the RI of the particles. The polymer contribution is accounted by multiplication of the RI difference $(\Delta n)$ between particles and surrounding polymer with the square of $V_{\mathrm{p}}$. Since $V_{\mathrm{p}}$ is $<1, V_{\mathrm{p}}^{2}$ is even lower than $V_{\mathrm{p}}$. Thus, this equation gives more weight to the contribution of the particles than the polymer matrix.

\subsection{Transparent Polymer Nanocomposites at Different Levels of Loading}

The most significant advantage of using nanosized particles is that blending of a small quantity of particles into the bulk of a polymer can drastically alter the photo physical properties of the latter. For example, Li et al reported a transparent PMMA/ZnO nanocomposite that showed significant protection against UV light at very low particle content. A ZnO particle content below 0.11 wt\% exhibited very effective UV shielding over the full UV range. ${ }^{[13 \mathrm{~b}]}$ This performance was proposed to be the result of a homogeneous distribution of the nanoparticles which were said to have $2.8 \pm 0.4 \mathrm{~nm}$ in diameter in the PMMA matrix. A low particle content is also prone to achieve strong luminescence. ${ }^{[105]}$ Transparent and luminescent nanocomposites were prepared from manganese doped $\mathrm{ZnS}$ particles of $22 \mathrm{~nm}$ in diameter dispersed in PMMA at a load of $1.1 \mathrm{wt} \%{ }^{[38 \mathrm{a}]}$ These nanoparticles gave a very strong orange emission with a quantum yield of $29.8 \%$ when irradiated with light of $331 \mathrm{~nm}$ wavelength. The emission features of the nanoparticles remained unaffected by in situ free radical polymerization. This supports the findings mentioned earlier in this review that the, free radicals which are present during the in situ polymerization do not alter the surface and/or internal microstructure of the particles present in the polymerization medium.

Following the current literature, the highest content of inorganic particles for which transparency is still maintained lies in range of 10-15 wt\%, in the particular case of PMMA as the matrix. The loading level varies for different particle/surfactant systems, for example, $10 \mathrm{wt} \%$ for methylphenylsiloxane (MPS)-modified $\mathrm{TiO}_{2}$ particles with $15 \mathrm{~nm}$ of diameter ${ }^{[106]}$ and $11 \mathrm{wt} \%$ for $t \mathrm{BuPO}_{3} \mathrm{H}_{2}$-modified ZnO nanoparticles with $22 \mathrm{~nm}$ diameter. ${ }^{[58,63 \mathrm{~b}]}$ When the size of the particles is reduced to $4 \mathrm{~nm}$ as in the case of MPSmodified $\mathrm{ZrO}_{2}, 15 \mathrm{wt} \%$ can be loaded into the polymer matrix. ${ }^{[107]}$ It is believed that increasing the particle content beyond this range leads to strong flocculation and therefore opacity. However, under special conditions a much higher particle content can be achieved while still maintaining transparency. For instance, Yang and co-workers ${ }^{[36 b]}$ reported a transparent nanocomposites based on a copolymer of $N, N$-dimethylacrylamide (DMAA) and styrene loaded with up to $30 \mathrm{wt} \%$ of $\mathrm{ZnS}$ nanoparticles. The diameter of ZnS nanoparticles was said to be in the range between 2 and $4 \mathrm{~nm}$. The particles were stated to be individually dispersed in the polymer matrix and the bulk nanocomposite had good light transmittance in the visible. One is tempted to assume that the size of the $\mathrm{ZnS}$ nanoparticles is comparable with the size of a polymer segment and this fact plays a key role in construction of homogeneous dispersion of the nanoparticles in the polymer matrix. Based on a rough calculation, the size of the polymer statistical segment is approximated to be about $10 \mathrm{~nm}^{3}$, therefore the polymer segment does not "see" the particles as a different phase and a potential depletion flocculation is prevented on account of the miscibility between polymer segments and objects of the same size. In addition to homogeneous particle dispersion, proper selection of material components in terms of RI also contributes to transparency. The same authors estimated the RI of ZnS nanoparticles to be approximately 1.80 . This value is significantly lower than the one of bulk ZnS $(n=2.37)$ and was accounted for by the large contribution of the disordered surface layer to the bulk properties of the very small particles. Both comonomers have a comparatively large RI for organic compounds $\left(n_{\mathrm{DMAA}}=1.47\right.$ and $\left.n_{\text {styrene }}=1.59\right)$. The combination of these materials minimizes somewhat the RI mismatch between particles and matrix and improves transparency accordingly. It would be informative to compare the transparency of this nanocomposite with a composite prepared with the same particles in a commodity plastic such as PMMA.

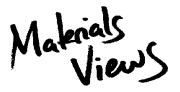

www.MaterialsViews.com 
It is widely accepted that there is a trade-off between particle content and transparency. In contrast to common belief, an improvement in transmission can still be achieved when the particle content increases. Tsuzuki ${ }^{[108]}$ recently reported transmission of composites films of $\mathrm{ZnO}$ particles dispersed in a capric triglyceride matrix at different particle contents. The particles had been coated with poly(methylsilsesquioxane) and their diameter was $32 \pm 8 \mathrm{~nm}$ on average. Transmission of a composite film with 60 wt\% ZnO particles was found to be almost the same as that of a film containing $35 \mathrm{wt} \%$. The authors considered this unexpectedly high transmittance as "abnormal" in the sense that a saturation of transmission to a near-constant value was observed even when the particle content was increased to $60 \%$. The improvement in transmittance cannot be explained by Rayleigh scattering, which is only applicable to dilute systems containing spatially uncorrelated particles. However, the high transmittance must be attributed to coherent forward scattering owing to a periodic internal structure, namely uniform packing of the particles throughout the polymer matrix. If the particles are spaced out regularly and the particle size becomes comparable with the interparticle distance, the waves scattered from each individual particle interfere destructively in all directions except for that of the incident beam and a few other directions for which (at fixed wave length) Bragg-type scattering is allowed. Moreover, multiple light scattering, which is commonly observed in highly disordered systems, is also suppressed. The incident light passes through such material in selected directions with very low transmission loss. This is not a novel finding at all. It seems worth mentioning that in earlier reports high transmittance at high loadings was shown for a technically relevant model heterogeneous latex/poly(vinyl chloride) (PVC) system. Figure 19a shows the scattering profiles of this system as a function of scattering angle at different contents of latex particles. It is obvious that as the particle content increases, the maxima of the scattering curves get sharper. Moreover, a strong ring-like pattern becomes evident in the diffraction profile (inset of the Figure 19a) indicating the development of more ordered and periodic arrangement of the latex particles in the PVC matrix. Optical micrographs which are shown in panel b of Figure 19 also support this argument. The micrographs reveal the state of dispersion of the latex particles in PVC at 7 and $60 \mathrm{vol \% .}$ A highly ordered and periodic arrangement of the latex particles in the PVC matrix is apparent at $60 \mathrm{wt} \%$ where the sample exhibits very high optical clarity. ${ }^{[09]}$

\subsection{Transparency by Index Matching}

Homogeneous dispersion of nanosized particles smaller than the wavelength of incident light has been considered to be the main requirement to attain optical clarity in

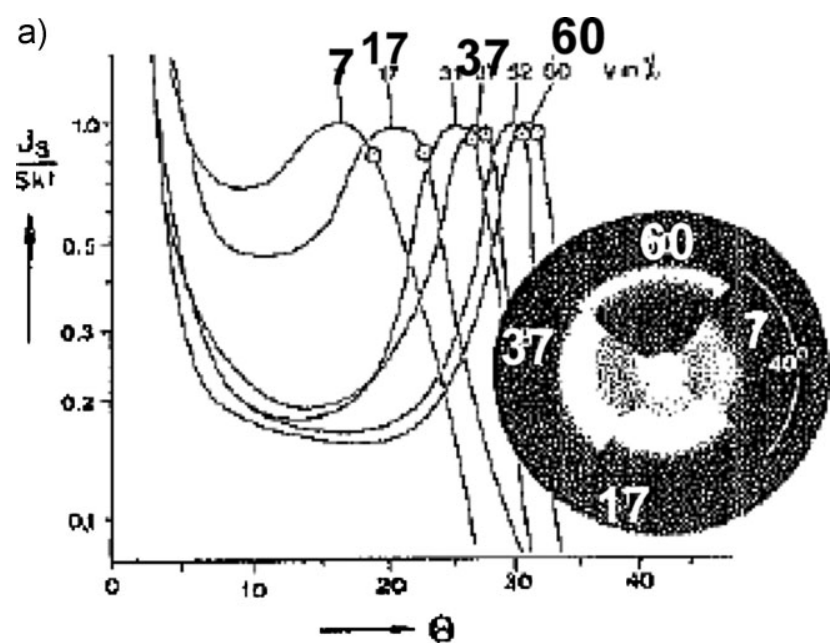

b)
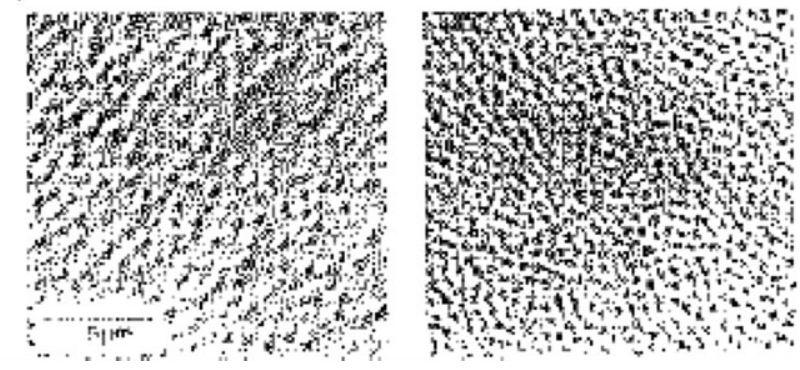

Figure 19. (a) Scattering profiles and diffraction pattern of PVCfilms loaded with latex particles. (b) Optical micrographs of the films at a loading of 7 and 60 vol\%. Showing the increase in order at increasing loading level. Data reprinted from ref. ${ }^{[109]}$ with permission of $\mathrm{G}$. Thieme Publishers.

polymer/inorganic nanoparticle composites. Although the scattering of the incident light is minimized under such circumstances, this requirement cannot guarantee transparency. Significant scattering will still arise from even small particles individually dispersed in polymers due to RI mismatch between particles and surrounding polymer matrix. Index matching among the material components (between particles and polymer) can be an efficient approach to suppress light scattering and improve the transparency. This essentially means that the incoming light cannot distinguish the particles from the matrix; hence it can propagate through the material unscattered. In essence, proper selection and design of the components can lead to index matching. To this aim, inorganic pigment particles are covered by a lower RI material such that the RI of this colloidal nanocomposite particle as a whole is reduced to the level of the surrounding matrix. In the following, we cite recent examples employing this approach. Bombalski et al. ${ }^{[110]}$ have demonstrated that the scattering of $\mathrm{SiO}_{2}$ particles in toluene can efficiently be suppressed by grafting PS chains from the particle surface with appropriate composition, molecular weight, and graft 
density. The choice of chemistry of core and shell is critical such that the effective RI of entire colloidal composite matches to the one of a given polymer matrix. How much thickness is required for index matching can be estimated by Maxwell-Garnett theory for each given diameter of the core particle. A significant reduction (approximately two orders of magnitude) in the scattering intensity was observed for a sample in which the index matching condition was satisfied as compared to neat particles. This event is called as null-scattering by the authors. It must be noted that these results refer to particles dispersed in a lowmolecular-weight liquid. Likewise, Parlak and Demir ${ }^{[111]}$ reported the synthesis of hybrid particles with $\mathrm{CeO}_{2}$ core and a PMMA shell. The particles were blended with a transparent PS matrix and transparency of the ternary composite system was examined. It was shown that null scattering situation can be reached in the solid state, as well. Moreover, it needs mentioning that index matching is not possible for all wavelengths of light simultaneously because the dispersion of RI is quite different for organics and inorganics. Organics tend to have a much steeper dependence of the RI on wavelength compared to common inorganics. Fu et al employed the same strategy for core/ shell-type silica/titania particles and reported a high transmittance when they were dispersed into an epoxy matrix. Titania is one of the metal oxides with very high index (2.50). ${ }^{[112]}$ Silica $(n=1.44)$, on the other hand, was used to reduce the overall RI to the level of that of the surrounding epoxy matrix $(n=1.54)$. The nanocomposites with the $1 \mathrm{wt} \%$ of core/shell particles with shell thickness as a variable are presented in Figure 20. An epoxy nanocomposite prepared with merely core silica nanoparticles without a titania shell even at a loading of $1 \mathrm{wt} \%$ is totally opaque. However, the transparency of the composites varies as the titania shell thickness changes. Once the thickness is reached to the value that satisfies index matching, an almost transparent nanocomposite material is obtained although the particle content remains unchanged. Further increase in shell thickness deteriorates transparency and turbidity reappears at $60 \mathrm{wt} \%$ coverage

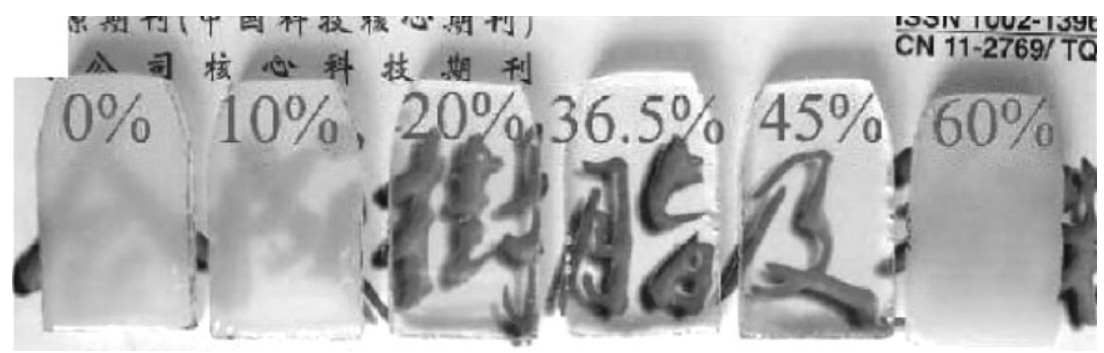

Figure 20. Micrographs indicating the transmissive properties of epoxy-resins blended with $\mathrm{TiO}_{2} @ \mathrm{SiO}_{2}$ (core/shell) nanoparticles at different thicknesses of the $\mathrm{TiO}_{2}$ shell. When index matching between particles and matrix is reached, optimal transmission is observed. Data reprinted from ref. ${ }^{[112]}$ with permission of ACS. by titania. If the thickness of the shell increases over what is required for index matching, scattering will reappear.

In addition to core/shell type nanostructured particles, binary mixtures of metal oxide particles have been used as well. Silica fillers are widely used in composite structures because of their well-studied silanization mechanisms resulting in excellent polymer/filler particles. It is also a good host for heavy-metal centers. It has no absorption band at all in the visible spectrum and has RI at 1.46 at $633 \mathrm{~nm}$. The addition of heavy metals into $\mathrm{SiO}_{2}$ structures such as $\mathrm{Ta}_{2} \mathrm{O}_{5}{ }^{[113]}$ or $\mathrm{ZnO}^{[114]}$ allows to control the RI of the solid mixture. For the $\mathrm{SiO}_{2} / \mathrm{Ta}_{2} \mathrm{O}_{5}$ system, $\mathrm{RI}$ was increased from 1.46 to more than 1.8 .

\section{Conclusion and Outlook}

A critical review of the recent literature on the topic of blends of nanoparticles with amorphous polymers reveals the inherent difficulties of the subject. First of all, very few materials are commercially or at least insufficient quantities available in form of reasonably well defined nanoparticles to allow quantitative studies on formation and properties of their blends with polymers. Obvious questions ask for the properties of the blends depending on particle size, size distribution, and surface chemistry of the particles. Secondly, the tendency of nanoparticles to form aggregates hampers all attempts to create homogeneous distributions of particles in a given polymer matrix although the latter would be the ultimate goal to test performance against theories which have evolved in the recent years. Furthermore, one needs to differentiate between chemically inert particles ("fillers") and chemically reactive solid additives. $\mathrm{CB}$, fullerenes and CNT are examples for the latter. Specifically $\mathrm{CB}$ is a long and heavily investigated nanomaterial with immensely important application in terms of its blends with rubber.

Interestingly enough, even "chemically inert" particles have a profound effect on the kinetics of free radical bulk polymerization, given that the specific surface of the particles surmounts a critical value. Thus, the presence of nanoparticles of, for example, ZnO or AlN suppresses the gel effect in free radical bulk polymerization of methyl methacrylate as an example. Although the particles are individually dispersed in the monomer they undergo flocculation and form clouds in the bulk polymer. This is a well-known phenomenon in colloid science and bears the name of "depletion flocculation," a consequence of entropic interaction of polymer chains and particles. Whether this flocculation can be suppressed making

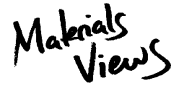

www.MaterialsViews.com
Macromol. Mater. Eng. 2012, 297, 838-863

(c) 2012 WILEY-VCH Verlag GmbH \& Co. KGaA, Weinheim 
the particles so small that they approach the size of the constitutive ("repeat") unit of the polymer or at least that of its "statistical element" remains an open question. In any case, it remains a major challenge to prepare stable and handable particles of that small size in sufficient quantities. The volume of those particles should be order of 1-2 $\mathrm{nm}^{3}$ meaning that their specific surface would fall into the range of typically $1000 \mathrm{~m}^{2} \cdot \mathrm{g}^{-1}$ ! The surface chemistry of the particles needs to be adjusted to the properties of the polymer matrix in order to achieve compatibility. Compatibility is here understood as the aptitude of the polymer segment to "wet" the particle surface. If wetting is not possible, mixing of polymer segments and particles cannot be achieved.

This or similar considerations have triggered many attempts to make use of surfactants for the solid particles to achieve compatibility. Moreover, surfactants which expose polymerizable groups have been used to counteract diffusion and, thereby flocculation of the particles in bulk polymerization of monomer/particle mixtures. The surfactant loaded particles become centers of crosslinks with very high functionality in the process of polymerization. The presence of surfactants adds a further component to the blend which contributes significantly to the overall performance of the material. This is particularly true for nanosized particles for which a full coverage of the surface implements a volume fraction of surfactants easily equal or even larger than the volume fraction of the particles alone.

Since the main reason to make use of surfactants is to adjust the surface free energy of the particles to that of the polymer matrix and thus induce wetting and suppress particle/particle clustering it becomes a goal to develop synthetic procedures which allow manufacturing nanoparticles that are surface-capped by organic species. These species have a double function: first, they stabilize the nanoparticles against Ostwald-ripening and other processes leading to increase of the size and shape of the particles while they are prepared, processed and stored; second they reduce the surface free energy of the particles to that of the polymer which will be the matrix for dispersion.

A further set of open questions concerns the study of optical properties of the blends. Here, the dependence of transmission, gloss, haze and translucency on particle concentration, particle topology, and state of order (e.g., "randomness") are important tasks with considerable impact and practical applications. While scattering methods seem to provide the obvious tools to quantify the topology of the particle dispersion this is by no means a simple task considering light scattering for reasons of multiple scattering processes and the contribution of density fluctuations that result in RI fluctuations, imposed by the particles on the surrounding bulk polymer. In other words, simple additivity rules may not be valid to allow the decomposition of signals extracted from the response of the blend to exposure to electromagnetic radiation. Similar considerations would be valid for dielectric or other relaxation spectroscopies. Clearly, physically meaningful mixing rules need to be developed which have a predictive power for the performance of polymer/nanoparticle blends.

In summary, the promise that blends of polymers and nanoparticles may open new opportunities for the design of novel materials remains to be tested against experimental evidence beyond of what is already state-of-the-art, for example, for blends of $\mathrm{CB}$ and rubber or pigmented coatings. The biggest challenge is seen for the design and realization of processes by which nanoparticles can be produced in sufficient quantities and reproducible quality to be blended with polymers. The number of available methods and materials remains still quite scarce given the quality one likes to see in experimentation.

\section{Nomenclature}

$\begin{array}{ll}\text { AIBN } & \text { azoisobutyronitrile } \\ \text { ATRP } & \text { atom-transfer radical polymerization } \\ \text { BPO } & \text { dibenzoyl peroxide } \\ \text { DLS } & \text { dynamic light scattering } \\ \text { DMAA } & \text { N-dimethylacrylamide } \\ \text { DMAEMA } & \text { dimethylaminoetyl methacrylate } \\ \text { DSC } & \text { differential scanning calorimetry } \\ \text { EDMA } & \text { ethylene dimethacrylate } \\ \text { EPR } & \text { electron paramagnetic resonance } \\ \text { IR } & \text { infrared } \\ \text { LCD } & \text { liquid crystal display } \\ \text { LMA } & \text { lauryl methacrylate } \\ \text { MMA } & \text { methyl methacrylate } \\ \text { MWCNT } & \text { multi-walled carbon nanotube } \\ \text { NP } & \text { nanoparticle } \\ \text { OVDAC } & \text { octadecyl-p-vinylbenzyldimethylammonium } \\ & \text { chloride } \\ \text { PHEMA } & \text { poly(hydroxy ethylmethacrylate) } \\ \text { PMMA } & \text { poly(methyl methacrylate) } \\ \text { PRISM } & \text { polymer reference interaction site model } \\ \text { PS } & \text { polystyrene } \\ \text { PSD } & \text { particle size distribution } \\ \text { PU } & \text { polyurethane } \\ \text { PVC } & \text { poly(vinyl chloride) } \\ \text { RI } & \text { refractive index } \\ \text { SANS } & \text { small angle neutron scattering } \\ \text { SAXS } & \text { small angle X-ray scattering } \\ \text { SEM } & \text { scanning electron microscopy } \\ \text { SWCNT } & \text { single wall carbon nanotube } \\ \text { TBAc } & \text { tetrabutylammonium acetate } \\ \text { BBuPO }{ }_{3} \mathrm{H}_{2} & \text { tert.-butylphosphonic acid } \\ \text { TEM } & \text { transmission electron microscopy } \\ \text { TEOS } & \text { tetraethoxysilane } \\ & \end{array}$


$T_{\mathrm{g}} \quad$ glass transition temperature

UV ultraviolet

Acknowledgements: M. M. D. acknowledges the financial support from the Scientific and Technological Research Council of Turkey (TUBITAK) project encoded TBAG-109T905.

Received: March 14, 2012; Published online: June 18, 2012; DOI: 10.1002/mame.201200089

Keywords: bulk polymerization; nanocomposites; particle aggregation; radical polymerization; transparency

[1] a) K. I. Winey, R. A. Vaia, MRS Bull. 2007, 32, 314; b) R. A. Vaia, J. F. Maguire, Chem. Mater. 2007, 19, 2736.

[2] a) A. Usuki, Y. Kojima, M. Kawasumi, A. Okada, Y. Fukushima, T. Kurauchi, O. Kamigaito, J. Mater. Res. 1993, 8, 1179; b) Y. Kojima, A. Usuki, M. Kawasumi, A. Okada, Y. Fukushima, T. Kurauchi, O. Kamigaito, J. Mater. Res. 1993, 8, 1185.

[3] a) H. Althues, J. Henle, S. Kaskel, Chem. Soc. Rev. 2007, 36, 1454; b) J. E. Mark, B. Erman, "Rubber Elasticity: A molecular Primer", 2nd edition, Cambridge University Press, Toronto 1988, p. 205.

[4] N. Tomczak, D. Janczewski, M. Y. Han, G. J. Vancso, Prog Polym. Sci. 2009, 34, 393.

[5] W. D. Kingery, H. K. Bowen, D. R. Uhlmann, "Introduction to Ceramics", 2nd edition, John Wiley \& Sons, New York 1960.

[6] T. Matsuura, H. Ikeda, N. Idota, R. Motokawa, Y. Hara, M. Annaka, J. Phys. Chem. B 2009, 113, 16314.

[7] K. M. Meek, D. W. Leonard, C. J. Connon, S. Dennis, S. Khan, Eye 2003, 17, 927.

[8] a) J. I. Clark, Exp. Eye Res. 2004, 78, 427; b) D. M. Maurice, J. Phys. 1957, 136, 263.

[9] T. Feuk, IEEE Trans. Biomed. Eng. 1970, BM17, 186.

[10] D. A. Stone, L. T. J. Korley, Macromolecules 2010, 43, 9217.

[11] a) M. Agrawal, S. Gupta, N. E. Zafeiropoulos, U. Oertel, R. Hassler, M. Stamm, Macromol. Chem. Phys. 2010, 211, 1925; b) B. J. Ash, R. W. Siegel, L. S. Schadler, Macromolecules 2004, 37, 1358; c) S. Vaudreuil, A. Labzour, S. Sinha-Ray, K. El Mabrouk, M. Bousmina, J. Nanosci. Nanotechnol. 2007, 7, 2349.

[12] a) P. Satraphan, A. Intasiri, V. Tangpasuthadol, S. Kiatkamjornwong, Polym. Adv. Technol. 2009, 20, 473; b) L. Chen, J. Zhu, O. Li, S. Chen, Y. R. Wang, Eur. Polym. J. 2007, 43, 4593; c) E. Ozkaraoglu, K. Tunc, S. Suzer, Polymer 2009, 50, 462 .

[13] a) A. H. Yuwono, B. H. Liu, J. M. Xue, J. Wang, H. I. Elim, W. Ji, Y. Li, T. J. White, J. Mater. Chem. 2004, 14, 2978; b) S. Li, M. S. Toprak, Y. S. Jo, J. Dobson, D. K. Kim, M. Muhammed, Adv. Mater. 2007, 19, 4347.

[14] M. M. Demir, R. Munoz-Espi, I. Lieberwirth, G. Wegner, J. Mater. Chem. 2006, 16, 2940.

[15] S. Gross, D. Camozzo, V. Di Noto, L. Armelao, E. Tondello, Eur. Polym. J. 2007, 43, 673.
[16] P. H. Huang, T. C. Huang, Y. T. Sun, S. Y. Yang, Opt. Express 2008, 16, 3041

[17] K. D. Singer, T. Kazmierczak, J. Lott, H. Song, Y. H. Wu, J. Andrews, E. Baer, A. Hiltner, C. Weder, Opt. Express 2008, 16, 10358.

[18] P. G. Ursell, D. J. Spalton, M. V. Pande, E. J. Hollick, S. Barman, J. Boyce, K. Tilling, J. Cataract Ref. Surg. 1998, 24, 352.

[19] M. K. Szczurowski, T. Martynkien, G. Statkiewicz-Barabach, W. Urbanczyk, L. Khan, D. J. Webb, Opt. Lett. 2010, 35, 2013.

[20] O. Shen, Y. Cao, S. Liu, L. Gan, J. M. Li, Z. X. Wang, J. S. Hui, X. F. Guo, D. S. Xu, Z. F. Liu, J. Phys. Chem. Lett. 2010, 1 1269.

[21] L. W. Lin, T. K. Shia, C. J. Chiu, J. Micromech. Microeng. 2000, 10, 395.

[22] G. Allegra, G. Raos, M. Vacatello, Prog. Polym. Sci. 2008, 33, 683.

[23] H. Kim, A. A. Abdala, C. W. Macosko, Macromolecules 2010, $43,6515$.

[24] M. A. El-Sayed, Acc. Chem. Res. 2001, 34, 257.

[25] a) V. V. Vodnik, J. V. Vukovic, J. M. Nedeljkovic, Colloid Polym. Sci. 2009, 287, 847; b) Y. Liu, E. N. Mills, R. J. Composto, J. Mater. Chem. 2009, 19, 2704.

[26] P. Mulvaney, Langmuir 1996, 12, 788.

[27] a) H. Zou, S. S. Wu, J. Shen, Chem. Rev. 2008, 108, 3893; b) G. Michael, H. Ferch, "Grundlagen und Anwendung von Aerosol" in Schriftenreihe "Pigmente" des Geschäftsbereiches Anorganische Chemieprodukte der DEGUSSA, Frankfurt/Germany, 1993, p. 60 f.

[28] O. W. Yuan, J. E. Mark, Macromol. Chem. Phys. 1999, 200, 206.

[29] S. R. Raghavan, H. J. Walls, S. A. Khan, Langmuir 2000, 16, 7920.

[30] J. Nordstrom, A. Matic, J. Z. Sun, M. Forsyth, D. R. MacFarlane, Soft Matter 2010, 6, 2293.

[31] P. Cassagnau, Polymer 2008, 49, 2183.

[32] C. Parneix, J. Persello, R. Schweins, B. Cabane, Langmuir 2009, $25,4692$.

[33] S. M. Cho, W. Y. Choi, J. Photochem. Photobiol., A 2001, 143, 221.

[34] a) S. Tsunekawa, T. Fukuda, A. Kasuya, J. Appl. Phys. 2000, 87, 1318; b) Ö. Kepenekçi, M. Emirdag-Eanes, M. M. Demir, J. Nanosci. Nanotechnol. 2011, 11, 3565.

[35] a) M. J. F. Digonnet, "Rare-Earth-Doped Fiber Lasers and Amplifiers", 2nd edition, CRC Press, Boca Raton 2001; b) M. C. Tan, S. D. Patil, R. E. Riman, ACS Appl. Mater. Interfaces 2010, 2, 1884.

[36] a) C. Guan, C. L. Lu, Y. R. Cheng, S. Y. Song, B. Yang, J. Mater. Chem. 2009, 19, 617; b) Y. R. Cheng, C. Lu, Z. Lin, Y. F. Liu, C. Guan, H. Lu, B. Yang, J. Mater. Chem. 2008, 18, 4062; c) C. L. Lu, Y. R. Cheng, Y. F. Liu, F. Liu, B. Yang, Adv. Mater. 2006, 18, 1188; d) C. L. Lu, Z. C. Cui, Z. Li, B. Yang, J. C. Shen, J. Mater. Chem. 2003, 13, 526.

[37] H. Zhang, Z. C. Cui, Y. Wang, K. Zhang, X. L. Ji, C. L. Lu, B. Yang, M. Y. Gao, Adv. Mater. 2003, 15, 777.

[38] a) H. Althues, R. Palkovits, A. Rumplecker, P. Simon, W. Sigle, M. Bredol, U. Kynast, S. Kaskel, Chem. Mater. 2006, 18, 1068; b) H. Althues, P. Simon, S. Kaskel, J. Mater. Chem. 2007, 17, 758.

[39] D. V. Talapin, I. Mekis, S. Gotzinger, A. Kornowski, O. Benson, H. Weller, J. Phys. Chem. B 2004, 108, 18826.

[40] O. V. Vassiltsova, D. A. Jayez, Z. Y. Zhao, M. A. Carpenter, M. A Petrukhina, J. Nanosci. Nanotechnol. 2010, 10, 1635.

[41] X. Y. Oi, K. Y. Pu, C. Fang, G. A. Wen, H. Zhang, F. Y. C. Boey, O. L. Fan, L. H. Wang, W. Huang, Macromol. Chem. Phys. 2007, 208, 2007.

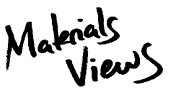


[42] T. Kyprianidouleodidou, W. Caseri, U. W. Suter, J. Phys. Chem. 1994, 98, 8992.

[43] D. V. Talapin, J. S. Lee, M. V. Kovalenko, E. V. Shevchenko, Chem. Rev. 2010, 110, 389.

[44] M. Tamborra, M. Striccoli, M. L. Curri, A. Agostiano, J. Nanosci. Nanotechnol. 2008, 8, 628.

[45] S. Y. Lu, M. L. Wu, H. L. Chen, J. Appl. Phys. 2003, 93, 5789.

[46] E. P. Pluddemann, "Silane Coupling Agents", 2nd edition, Plenum Press, New York 1991.

[47] O. Masala, R. Seshadri, Annu. Rev. Mater. Res. 2004, 34, 41.

[48] M. M. Demir, B. Altin, S. Özçelik, Compos. Interfaces 2010, 17, 831.

[49] C. P. Li, M. Akinc, J. Wiench, M. Pruski, C. H. Schilling, J. Am. Ceram. Soc. 2005, 88, 2762.

[50] V. Khrenov, M. Klapper, M. Koch, K. Mullen, Macromol. Chem. Phys. 2005, 206, 95.

[51] S. H. Stelzig, M. Mapper, K. Muellen, Adv. Mater. 2008, 20, 929.

[52] W. K. Wang, M. L. Zheng, W. O. Chen, F. Jin, Y. Y. Cao, Z. S. Zhao, X. M. Duan, Langmuir 2011, 27, 3249.

[53] J. N. Israelachvili, "Intermolecular and Surface Forces", Academic Press, London 1992.

[54] G. Wegner, M. M. Demir, M. Faatz, K. Gorna, R. Munoz-Espi, B. Guillemet, F. Grohn, Macromol. Res. 2007, 15, 95.

[55] C. Goebbert, M. A. Aegerter, D. Burgard, R. Nass, H. Schmidt, J. Mater. Chem. 1999, 9, 253.

[56] T. Siebel, Organokompatible Zinnoxid-Nanopartikel, Ph. D. thesis, Johannes Gutenberg University Mainz 2007.

[57] C. H. Hung, W. T. Whang, J. Mater. Chem. 2005, 15, 267.

[58] M. M. Demir, K. Koynov, U. Akbey, C. Bubeck, I. Park, I. Lieberwirth, G. Wegner, Macromolecules 2007, 40, 1089.

[59] C. Sanchez, B. Julian, P. Belleville, M. Popall, J. Mater. Chem. 2005, 15, 3559.

[60] J. Pyun, K. Matyjaszewski, Chem. Mater. 2001, 13, 3436.

[61] B. Lepoittevin, N. Pantoustier, M. Devalckenaere, M. Alexandre, D. Kubies, C. Calberg, R. Jerome, P. Dubois, Macromolecules 2002, 35, 8385.

[62] a) L. H. Sun, Z. G. Yang, X. H. Li, J. Appl. Polym. Sci. 2008, 107, 1842; b) M. A. Paul, M. Alexandre, P. Degee, C. Calberg, R. Jerome, P. Dubois, Macromol Rapid Commun. 2003, 24, 561.

[63] a) M. M. Demir, P. Castignolles, U. Akbey, G. Wegner, Macromolecules 2007, 40, 4190; b) M. M. Demir, M. Memesa, P. Castignolles, G. Wegner, Macromol. Rapid Commun. 2006, 27, 763.

[64] M. Arai, K. Arai, S. Saito, J. Polym. Sci. A1 1982, 20, 1021.

[65] J. P. Zheng, R. Zhu, Z. H. He, G. Cheng, H. Y. Wang, K. D. Yao, J. Appl. Polym. Sci. 2010, 115, 1975.

[66] J. Yang, T. Hasell, W. X. Wang, J. Li, P. D. Brown, M. Poliakoff, E. Lester, S. M. Howdle, J. Mater. Chem. 2008, 18, 998.

[67] E. Bourgeat-Lami, "Organic/Inorganic Composites by multiphase polymerization" in Dendrimers, Assemblies and Nanocomposites, Vol. 5 (Eds., R. Arshady, A. Guyot), Citus Books, London 2002, p. 149.

[68] R. Advincula, Adv. Polym. Sci. 2006, 197, 107.

[69] L. M. Clayton, A. K. Sikder, A. Kumar, M. Cinke, M. Meyyappan, T. G. Gerasimov, J. P. Harmon, Adv. Funct. Mater. 2005, 15, 101.

[70] C. C. Zeng, L. J. Lee, Macromolecules 2001, 34, 4098.

[71] L. J. Wang, S. P. Su, D. Chen, C. A. Wilkie, Polym. Degrad. Stab. 2009, 94, 770.

[72] E. Logakis, P. Pissis, D. Pospiech, A. Korwitz, B. Krause, U. Reuter, P. Potschke, Eur. Polym. J. 2010, 46, 928.

[73] M. Krajnc, U. Sebenik, Polym. Compos. 2009, 30, 1678.
[74] a) D. H. Hey, Discuss. Faraday Soc. 1953, 216; b) J. Ueda, H. Yamaguchi, K. Shirai, T. Yamauchi, N. Tsubokawa, J. Appl. Polym. Sci. 2008, 107, 3300; c) M. Hevy, M. Szwarz, J. Chem. Phys. 1954, 22, 1621.

[75] Z. J. Jia, Z. Y. Wang, C. L. Xu, J. Liang, B. O. Wei, D. H. Wu, S. W. Zhu, Mater. Sci. Eng. A 1999, 271, 395.

[76] W. T. Ford, T. D. Graham, T. H. Mourey, Macromolecules 1997, $30,6422$.

[77] H. Y. Xu, Y. Y. Cao, X. L. He, Y. F. Wu, Y. M. Zhang, C. F. Wu, J. Macromol. Sci. B 2009, 48, 1190.

[78] S. J. Park, M. S. Cho, S. T. Lim, H. J. Choi, M. S. Jhon, Macromol. Rapid Commun. 2003, 24, 1070.

[79] a) D. Baskaran, J. R. Dunlap, J. W. Mays, M. S. Bratcher, Macromol. Rapid Commun. 2005, 26, 481; b) G. O. Guo, D. Yang, C. C. Wang, S. Yang, Macromolecules 2006, 39, 9035.

[80] J. H. Fan, M. X. Wan, D. B. Zhu, B. H. Chang, Z. W. Pan, S. S. Xe, J. Appl. Polym. Sci. 1999, 74, 2605.

[81] B. Z. Tang, H. Y. Xu, Macromolecules 1999, 32, 2569.

[82] L. Macarie, G. Ilia, J. Appl. Polym. Sci. 2007, 104, 247.

[83] G. Odian, "Principals of Polymerization", 4th edition, John Wiley \& Sons, New Jersey 2004.

[84] a) A. K. Nikolaidis, D. S. Achilias, G. P. Karayannidis, Ind. Eng. Chem. Res. 2011, 50, 571; b) D. S. Achilias, A. K. Nikolaidis, G. P. Karayannidis, J. Therm. Anal. Calorim. 2010, 102, 451.

[85] A. Tsuchida, M. Suda, M. Ohta, T. Yamauchi, N. Tsubokawa, J. Polym. Sci. Polym. Chem. 2006, 44, 2972.

[86] T. Kashiwagi, A. Inaba, J. E. Brown, K. Hatada, T. Kitayama, E. Masuda, Macromolecules 1986, 19, 2160

[87] a) A. G. Camp, A. Lary, W. T. Ford, Macromolecules 1995, 28 , 7959; b) Y. H. Hou, M. O. Zhang, M. Z. Rong, Polym. Int. 2004, 53,944 .

[88] A. Anzlovar, Z. C. Orel, M. Zigon, Eur. Polym. J. 2010, 46, 1216.

[89] S. H. Li, J. Qin, A. Fornara, M. Toprak, M. Muhammed, D. K. Kim, Nanotechnology 2009, $20,6$.

[90] A. Moussaid, W. C. K. Poon, P. N. Pusey, M. F. Soliva, Phys. Rev. Lett. 1999, 82, 225.

[91] T. Kuhl, Y. O. Guo, J. L. Alderfer, A. D. Berman, D. Leckband, J. Israelachvili, S. W. Hui, Langmuir 1996, 12, 3003.

[92] M. M. Demir, Y. Z. Menceloglu, B. Erman, Macromol. Chem. Phys. 2006, 207, 1515.

[93] W. C. K. Poon, J. Phys.: Condens. Matter 2002, 14, R859.

[94] M. Saric, H. Dietsch, P. Schurtenberger, Colloid Surf. A 2006, 291, 110.

[95] H. Sugimoto, K. Daimatsu, E. Nakanishi, Y. Ogasawara, T. Yasumura, K. Inomata, Polymer 2006, 47, 3754.

[96] M. M. Demir, Y. Z. Menceloglu, B. Erman, Polymer 2005, 46, 4127.

[97] L. Zhao, Y. G. Li, C. L. Zhong, J. Chem. Phys. 2007, 126, 014906.

[98] A. Horta, M. A. Pastoriza, Eur. Polym. J. 2005, 41, 2793.

[99] F. M. Willmouth, "Transparency, Translucency and Gloss" in Optical Properties of Polymers, (Ed., G. H. Meeten), Elsevier Applied Science Publishers, London \& New York 1986.

[100] U. Ozgur, Y. I. Alivov, C. Liu, A. Teke, M. A. Reshchikov, S. Dogan, V. Avrutin, S. J. Cho, H. Morkoc, J. Appl. Phys. 2005, 98.

[101] Polymer Handbook, 3rd edition, (Eds., J. Brandrup, E. H. Immergut), John Wiley \& Sons, New York 1998.

[102] C. L. Lu, B. Yang, J. Mater. Chem. 2009, 19, 2884.

[103] Y. O. Rao, S. Chen, Macromolecules 2008, 41, 4838.

[104] R. J. Nussbaumer, W. R. Caseri, P. Smith, T. Tervoort, Macromol. Mater. Eng. 2003, 288, 44. 
[105] J. A. Paramo, Y. M. Strzhemechny, A. Anzlovar, M. Zigon, Z. C. Orel, J. Appl. Phys. 2010, 108, 023517.

[106] D. Koziej, F. Fischer, N. Kranzlin, W. R. Caseri, M. Niederberger, ACS Appl. Mater. Interfaces 2009, 1, 1097.

[107] Y. O. Hu, S. X. Zhou, L. M. Wu, Polymer 2009, 50, 3609.

[108] T. Tsuzuki, Macromol. Mater. Eng. 2008, 293, 109.

[109] H. Breuer, "Optisches Verhalten mehrphasiger Systeme" in Polymere Werkstoffe Chemie und Physik, Vol. 1, Thieme, Stuttgart, New York 1985, p 441.
[110] L. Bombalski, H. C. Dong, J. Listak, K. Matyjaszewski, M. R. Bockstaller, Adv. Mater. 2007, 19, 4486.

[111] O. Parlak, M. M. Demir, ACS Appl. Mater. Interfaces 2011, 3, 4306.

[112] Y. O. Li, S. Y. Fu, Y. Yang, Y. W. Mai, Chem. Mater. 2008, 20 , 2637.

[113] H. Schulz, L. Madler, S. E. Pratsinis, P. Burtscher, N. Moszner Adv. Funct. Mater. 2005, 15, 830.

[114] Y. Yang, Y. O. Li, S. Y. Fu, H. M. Xiao, J. Phys. Chem. C 2008, 112 , 10553. 